\title{
Transition from galactic to extragalactic cosmic rays
}

\author{
R. Aloisio ${ }^{\mathrm{a}, \mathrm{b}, *}$, V. Berezinsky ${ }^{\mathrm{b}}$, A. Gazizov $^{\mathrm{b}, \mathrm{c}}$ \\ ${ }^{a}$ INAF, Arcetri Astrophysical Observatory, I-50125 Arcetri (FI), Italy \\ ${ }^{b} I N F N$, National Gran Sasso Laboratory, I-67010 Assergi (AQ), Italy \\ ${ }^{c}$ DESY Zeuthen, Platanenallee 6, D-157 Zeuthen, Germany
}

\section{N'Abstract}

'The study of the transition between galactic and extragalactic cosmic rays can shed more light on the end of the Galactic cosmic rays spectrum and the beginning of the extragalactic one. Three models of transition are discussed: ankle, dip and mixed composition 'models. All these models describe the transition as an intersection of a steep galactic component with a flat extragalactic one. 'Severe bounds on these models are provided by the Standard Model of Galactic Cosmic Rays according to which the maximum acceleration energy for Iron nuclei is of the order of $E_{\mathrm{Fe}}^{\max } \approx 1 \times 10^{17} \mathrm{eV}$. In the ankle model the transition is assumed at the -ankle, a flat feature in the all particle spectrum which observationally starts at energy $E_{a} \sim(3-4) \times 10^{18} \mathrm{eV}$. This model needs a new high energy galactic component with maximum energy about two orders of magnitude above that of the Standard Model. - The origin of such component is discussed. As observations are concerned there are two signatures of the transition: change of 'energy spectra and mass composition. In all models a heavy galactic component is changed at the transition to a lighter or proton 'component. As a result the ankle model predicts a galactic Iron component at $E<5 \times 10^{18} \mathrm{eV}$, while both HiRes and Auger data show that at $(2-5) \times 10^{18} \mathrm{eV}$ primaries are protons, or at least light nuclei. In the dip model the transition occurs at the 'second knee observed at energy $(4-7) \times 10^{17} \mathrm{eV}$ and is characterized by a sharp change of mass composition from galactic Iron 'to extragalactic protons. The ankle in this model appears automatically as a part of the $e^{+} e^{-}$pair-production dip. The mixed composition model describes transition at $E \sim 3 \times 10^{18} \mathrm{eV}$ with mass composition changing from the galactic Iron to extragalactic 'mixed composition of different nuclei. In most mixed composition models the spectrum is proton-dominated and it better fits 'HiRes than Auger data. The latter show a steadily heavier mass composition with increasing energy, and we discuss the models 'which explain it.

Keywords: ultrahigh energy cosmic rays, galactic cosmic rays, cosmic ray theory, cosmic ray experiment PACS: 95.85.Ry, 96.40.-z, 95.85.Ry, 98.70.Sa

\section{Introduction}

A clear understanding of the provenance of Cosmic Rays (CR) at different energetic regimes, namely their galactic or extragalactic origin, is of paramount importance in unveiling the nature of the sources of these particles, in particular at the highest energies.

The observed energy spectrum of CR has an approximately power-law behavior for 11 orders of magnitude in energy with several features that can be linked with particles propagation and acceleration. This power-law behavior is most probably indicative of a power-law acceleration

\footnotetext{
*Corresponding author

Email addresses: aloisio@arcetri.astro.it (R. Aloisio) berezinsky@lngs.infn.it (V. Berezinsky), askhat.gazizov@desy.de (A. Gazizov)
}

spectra, while spectral features may be assigned to changes in the origin of particles, their propagation and acceleration.

We shall consider these features starting from the highest energies.

If primary particles are protons, their spectrum must show a steepening which begins at $E_{\mathrm{GZK}} \sim 50 \mathrm{EeV}$. It is the famous Greisen 11, Zatsepin and Kuzmin [2] cutoff, referred to as GZK, caused by pion photo-production energy loss in collisions of protons with the Cosmic Microwave Background (CMB) photons. Most probably this cutoff is already observed in the HiRes (High Resolution Fly's Eye) [3] and TA (Telescope Array) [4] data, while the spectrum steepening observed by the PAO (Pierre Auger Observatory) [5] does not agree well, according to our calculations, with the predicted GZK shape and position.

An alternative to the protons as primaries is given by 
nuclei. Propagating through astrophysical backgrounds nuclei lose their energy in photo-disintegration and pair production processes, and also due to adiabatic expansion of the universe. The steepening of nuclei spectra due to photo-disintegration in the interactions with the CMB has been shortly mentioned by Greisen [1] and later considered quantitatively in [6, 7, 8, 9, 10]. The beginning of the steepening in this case (nuclei in the $\mathrm{CMB}$ radiation only) corresponds to the intersection of $e^{-} e^{+}$and adiabatic energy losses curves [7, 8]. The beginning of this steepening is lower than in the case of GZK cutoff and the shape of this feature differs from that of GZK. Recent accurate calculations show that the PAO spectrum steepening may be explained not only by nuclei interactions. It may be also produced as a result of a decreasing acceleration efficiency in the vicinity of the maximum energy that sources can provide $\left(E_{\max }\right)$ (for reviews see [1] $)$. The HiRes and TA data, in favor of proton composition, and the PAO data, in favor of nuclei composition, are further discussed in sections 3.2 and 3.3

There is another feature in the spectrum of UHE extragalactic protons, the pair-production dip [12], which, as in case of GZK cutoff, can be directly linked to the interaction of CRs with the CMB. This dip arises due to electron-positron production energy loss by extragalactic protons interacting with the CMB photons:

$$
p+\gamma_{\mathrm{CMB}} \rightarrow p+e^{+}+e^{-} \text {. }
$$

This feature has been studied in [13, 14, 15, 16]. The dip was observed with a very good statistical significance, $\chi^{2} /$ d.o.f. $\sim 1$, by the Fly's Eye, Yakutsk, Akeno-AGASA and HiRes detectors [17], but it is absent in the Auger data (see section 3.3 for a more detailed analysis).

The pair-production dip and GZK cutoff are signatures of protons. A confirmation of the shape of these features can be considered as an indirect evidence for a protondominated composition of primary CRs. For nuclei as primaries the shapes of the dip and cutoff are strongly different.

A different explanation of the dip has been proposed by Hill and Schramm [18]. They interpreted the dip observed in 1980s in terms of a two-component model; the low energy component was either galactic or produced by Local Supercluster. A similar model was later considered in [19]. The Hill-Schramm's interpretation is widely adopted now.

From 1960s there was observed a flattening in the Ultra High Energy Cosmic Rays (UHECR) spectrum, which was called the ankle. Discovery of this feature at the Volcano Ranch detector [20] was interpreted as the transition from a steep galactic component to a flatter extragalactic one. The ankle is clearly seen in the PAO data at $E_{a}^{\text {obs }} \approx 4 \mathrm{EeV}$ [21]. In [22] - 23] the ankle is considered as a feature where the transition from the galactic to extragalactic cosmic rays occurs. In the dip model [14, 15, 16] the ankle appears automatically as a part of the pair-production dip.

Below the ankle, at $E_{\text {skn }} \approx(0.4-0.7) \mathrm{EeV}$, there is a faint feature in the spectrum [24] called the second knee.
It is seen in many experiments (for a review see [25]). This feature is also often interpreted as the place of transition from galactic to extragalactic CRs, with the dip model [14, 15, 16] being among these works.

One more well known feature, the proton knee, occurs at $E_{p} \sim 4 \mathrm{PeV}$. It was discovered by the MSU (Moscow State University) array in 1958 [26]. The knee is most probably explained by the maximum acceleration energy for protons in the Galaxy. In the framework of the rigidity-dependent acceleration model it was predicted that the Iron knee is located at $Z_{\mathrm{Fe}}=26$ times higher energy, $E_{\mathrm{Fe}} \simeq 0.1 \mathrm{EeV}$. This feature was confirmed by the KASCADE Grande [27], so that the end of the Galactic CR spectrum is naturally connected with the Iron knee.

Based on the observational features described above, three models of the transition from galactic to extragalactic cosmic rays have been proposed.

In the ankle models [22] - 23] it is assumed that the transition occurs at the flat part of the observed spectrum in the energy interval $E_{a}^{\text {trans }} \sim(3-10)$ EeV. The transition energy is given by the intersection of a flat extragalactic spectrum and a very steep galactic one. In the majority of ankle models the extragalactic component is assumed to be pure proton, while the galactic one should be naturally represented by Iron nuclei at energies above the Iron knee. These models predict a transition from an Iron-dominated composition to a proton-dominated one at the ankle energy.

In the mixed composition model 28] it is assumed that the extragalactic component consists of nuclei of various types. Thus transition here occurs from Iron to lighter nuclei of mixed composition; it can occur at the ankle or nearby it.

In the dip model the transition begins at the second knee and is completed at the beginning of the dip, at $E \approx$ $1 \mathrm{EeV}$. The ankle in this model appears as an intrinsic part of the dip. Like in the ankle model, the transition here also occurs as an intersection of the flat extragalactic component (this flatness is especially prominent in the case of diffusive propagation) with a steep Galactic spectrum. In contrast to the ankle and mixed composition models, the dip model predicts an almost pure proton composition above $E \approx 1 \mathrm{EeV}$ and a pure Iron composition below this energy.

In this review we shall discuss recent modifications of these three basic models. The paper is organized as follows: in section 2 we briefly discuss the standard model of Galactic cosmic rays, focusing in particular on the end of the spectrum, in section 3 we discuss the spectra of cosmic rays at ultra high energy, focusing on their extragalactic origin, in section 4 we analyze the models of the transition from Galactic to extragalactic cosmic rays. Finally, in section 5 we shall present our conclusions. 


\section{The end of the Galactic CRs}

For a long time it was assumed that Supernova (SN) explosions may provide the observed cosmic rays. Baade and Zwicky [29] in 1934 were the first who understood that SNe energy release can produce enough energy to fill the Milky Way with the observed cosmic rays. Arguing with the adherents of extragalactic origin of the observed cosmic rays, Ginzburg and Syrovatsky put forward a model [30] of Galactic Cosmic Rays (GCR). Its basic elements were particle acceleration by SN shocks followed by a diffusive propagation in the Galactic magnetic fields, until they leave the Galaxy.

\subsection{Standard Model for Galactic Cosmic Rays (SM GCR)}

In spite of some small disturbing contradictions that will be discussed later, one may claim now that the Standard Model for Galactic Cosmic Rays exists (see reviews [31, 32, 33]). It is based on the Supernova Remnant (SNR) paradigm and includes four basic elements:

1. supernova remnants (SNRs) as sources;

2. SNR shock acceleration;

3. rigidity-dependent injection as a mechanism providing the observed CR mass composition, and

4. diffusive propagation of CRs in the Galactic magnetic fields.

Let us discuss these elements in more detail.

1. SNRs are able to provide the observed CR energy production in the Galaxy, which can be found [31] as

$$
Q_{\mathrm{cr}} \approx \omega_{\mathrm{cr}} c M_{g} / x_{\mathrm{cr}},
$$

where $\omega_{\text {cr }} \approx 0.5 \mathrm{eV} / \mathrm{cm}^{3}$ is the observed CR energy density, $c$ is the velocity of CR particle, $M_{g} \approx 5 \times 10^{42} \mathrm{~g}$ is the total mass of galactic gas, and $x_{\mathrm{cr}} \approx 7 \mathrm{~g} / \mathrm{cm}^{2}$ is the grammage traversed by $\mathrm{CR}$ before escaping the Galaxy. Using these numbers one obtains $Q \approx 2 \times 10^{40} \mathrm{erg} / \mathrm{s}$, which is less than $10 \%$ of the energy release in the form of kinetic energy of the SN ejecta per unit time.

2. The most efficient mechanism of diffusive shock acceleration (DSA) in SNRs was discovered in 1977 - 1978 [34]. A great progress has been reached during the last decade in the theory of acceleration. It was shown that cosmic ray streaming instability strongly amplifies magnetic fields upstream. It creates a highly turbulent field with strength up to $\delta B \sim B \sim 10^{-4} \mathrm{G}$ [35] (for recent works see [36]). At each moment of the shock propagation only particles accelerated up to maximum energy $E_{\max }$ can escape the acceleration region [37]. As a byproduct, this solves the problem of adiabatic energy losses, since particles escaping with $E_{\max }$ do not dwell long in the expanding SN shell.

$E_{\max }$ reaches the highest value at the beginning of the Sedov phase and then diminishes due to the shock deceleration. At each moment $t$ the spectrum of escaping particles has a narrow peak at $E_{\max }(t)$, but the spectrum integrated over time acquires a classical $E^{-2}$ shape with a flattening at highest energies 37. The maximum acceleration energy estimated in the Bohm regime of diffusion is given by

$$
E_{\max }=4 Z \frac{B}{10^{-4} \mathrm{G}}\left(\frac{W_{51}}{n_{g} / \mathrm{cm}^{3}}\right)^{2 / 5} \mathrm{PeV},
$$

where $B$ is the amplified magnetic field, $W_{51}$ is the kinetic energy of the shell in units $10^{51} \mathrm{erg}, n_{g}$ is the upstream density of the gas and $Z$ is the charge number of accelerated nuclei. Thus for protons and Iron nuclei maximum energies are

$$
\begin{aligned}
& E_{p}^{\max }=4 \times B_{-4} \mathrm{PeV} \\
& E_{\mathrm{Fe}}^{\max } \simeq 0.1 \times B_{-4} \mathrm{EeV}
\end{aligned}
$$

Here $E_{p}^{\max }$ describes well the position of the proton knee and $E_{\mathrm{Fe}}^{\max }$ predicts the position of the Iron one.

An important result on the structure of the knee was obtained in the KASCADE-Grande experiment [27]. The spectra were measured separately for electron-rich showers, initiated by protons and light nuclei, and electronpoor showers, corresponding to the heaviest nuclei of the Iron group. The steepening in the spectrum of electronpoor showers was found at $E \approx 80 \mathrm{PeV}$. With electronrich showers knee at $(3-5) \mathrm{PeV}$, the heaviest nuclei knee corresponds to Iron nuclei, as expected in the rigiditydependent acceleration model.

3. Observations show that nuclei in cosmic rays are systematically more abundant than in the interstellar medium of the solar neighborhood [38, 39, 40]; this occurs due to the injection of particles in the regime of acceleration [38, 39, 40]. It can be illustrated by the following simple consideration [16]. A particle $i$ from downstream $(i=A, p)$ can cross the shock and thus may be injected in the regime of acceleration if its Larmor radius $r_{L}(p) \geq d$, where $d$ is the thickness of the shock front. It readily gives the relation between a nucleus and proton injection momenta

$$
p_{\mathrm{inj}}^{A}=Z e B d / c=Z p_{\mathrm{inj}}^{p} .
$$

Therefore, $v_{\text {inj }}^{A}<v_{\text {inj }}^{p}$, which provides a higher injection rate for nuclei.

The same result may be also obtained in a more formal way. Consider a flux of accelerated particles $i, J_{i}(p)=$ $K_{i}\left(p / p_{\text {inj }}^{i}\right)^{-\gamma_{g}}$. Normalizing $J_{i}(p)$ by the condition

$$
\frac{4 \pi}{c} \int_{p_{\mathrm{inj}}^{i}}^{\infty} J_{i}(p) d p=\eta_{i} n_{i},
$$

where $n_{i}$ is the density of gas $i$ and $\eta_{i}$ is the fraction of this density injected into acceleration process, one obtains a ratio of nuclei and proton CR fluxes

$$
\frac{J_{A}(p)}{J_{p}(p)}=Z^{\gamma_{g}-1} \frac{\eta_{A} n_{A}}{\eta_{p} n_{p}} .
$$


Thus, the fraction of nuclei is enhanced by a factor $Z \eta_{A} / \eta_{p}$. For numerical calculations of CR nuclei abundances see [38, 39, 40].

4. CRs propagate in the Galaxy diffusively; they scatter off small-scale magnetic turbulence which may be described as a superposition of MHD waves with different amplitudes and random phases. This process is considered (see e.g. [31]) in the resonance approximation, when the particle giro-frequency is equal to the wave frequency in the system at rest with the particle motion along the average magnetic field. The magnetic field may be considered as a combination of an average (constant) field, $\vec{B}_{0}$, and a fluctuating component, $\vec{B}$. In 31] the parallel diffusion coefficient $D_{\|}(E)$ is calculated assuming $D_{\perp}(E) \ll D_{\|}(E)$ (see however numerical simulations [41] which do not support this assumption at the highest energies).

Proton and nuclei spectra, as calculated by Berezhko and Völk [42] within SM GCR, are shown in Fig. 1] in comparison with data. An agreement with observations is quite good at low energies, and the knee is confirmed at $E_{\mathrm{kn}} \approx 3 \mathrm{PeV}$ in proton and all-particle spectra. The Iron knee located at $E_{\mathrm{Fe}} \approx 80 \mathrm{PeV}$ is the most important prediction of the Standard Model; this feature was confirmed by the KASCADE-Grande detector [27]. The spectra beyond knees are expected to be very steep.

\subsection{Diffusive propagation: qualitative estimates}

Diffusive propagation is a phenomenon which currently provides problems to the Standard Model of Galactic CRs. The essence of these problems can be easily seen (for a detailed discussion see also the papers of other authors that cover these topics in the present topical issue).

The diffusion coefficient and its energy dependence are primarily determined by the turbulence spectrum $w(k)$, which in the most important cases is given in a power-law form $w(k) \propto k^{-m}$, where $k$ is the wave number. Then one has

$$
D(E) \propto E^{\mu}, \quad \mu=2-m,
$$

with $\mu=1 / 3, \quad \mu=1 / 2$ and $\mu=0$ for the Kolmogorov, Kraichnan and shock wave diffusion, respectively. In the Bohm regime with strong turbulence, the diffusion length is of the order of the gyroradius and $\mu=1$.

It is easy to verify that the space density $n_{p}$ of primary protons and nuclei is

$$
n_{p}(E) \propto E^{-\left(\gamma_{g}+\mu\right)} .
$$

It follows from $n_{p}(E) \sim Q_{p}(E) \tau_{\text {esc }}(E)$, where $Q_{p}(E) \propto$ $E^{-\gamma_{g}}$ is the generation (acceleration) rate of primaries, i.e. protons and nuclei, and $\tau_{\text {esc }}(E) \propto D(E)^{-1}$ is the escape time from the Galaxy. For non-relativistic diffusive acceleration $\gamma_{g}=2$; hence from the index of the observed spectrum, $\gamma_{g}+\mu=2.7$, one derives $\mu=0.7$. At very high energy this results in a too large anisotropy, $\delta(E) \propto D(E) \propto E^{\mu}$, and in a too small traversed grammage, $X_{\mathrm{cr}}(E) \propto 1 / D(E)$.
To solve the problem of small $X_{\mathrm{cr}}(E)$ it was suggested the effect of spallation inside CR sources and reacceleration by second order Fermi mechanism during CR propagation [43].

Concerning anisotropy, observations show its constant behavior with energy which is in contrast with the simple estimate above. This inconsistency is only apparent because the result $\delta(E) \propto D(E)$ refers to an average of the anisotropy amplitude computed over many source realizations, i.e. over a continuum of sources. In the framework of the SM for GCR, the actual value of anisotropy can be computed only taking into account the random nature (in space and time) of nearby recent SNRs 44.

It is easy to estimate the $e^{+} / e^{-}$ratio in the framework of SM and to compare it with the one measured in the PAMELA experiment [45].

The basic difference with protons and nuclei, considered above, is that the lifetime of HE electrons and positrons is much shorter than the escape time. It is given by energy losses due to synchrotron and inverse Compton radiation, $\tau_{e} \propto E^{-1}$. For primary HE electrons the rate of production (acceleration) is $Q_{e}(E) \propto E^{-\gamma_{g}}$ and the space density

$$
n_{e}(E) \sim Q_{e}(E) \tau_{e}(E) \propto E^{-\left(\gamma_{g}+1\right)} .
$$

Positrons in the SM GCR are produced as secondary particles in $p+p \rightarrow \pi^{+} \rightarrow e^{+}$interactions of CR protons with the interstellar gas. Using $n_{p}(E)$ from Eq.(9), one finds the rate of positron generation as

$$
Q_{e^{+}}(E) \sim n_{p}(E) \sigma_{p p} n_{\text {gas }} c \propto E^{-\left(\gamma_{g}+\mu\right)} .
$$

The space density of positrons $n_{e^{+}}(E)$ is then obtained as

$$
n_{e^{+}}(E) \sim Q_{e^{+}}(E) \tau_{e} \propto E^{-\left(\gamma_{g}+\mu+1\right)} .
$$

Thus in the framework of the SM of GCR the ratio

$$
n_{e^{+}} / n_{e-} \propto E^{-\mu}
$$

regularly diminishes with energy increasing. This simple estimate is in contrast with the observed increasing of the $e^{+} / e^{-}$ratio in the PAMELA data [45].

\subsection{Standard Model: Problems and Solutions}

The SM of Galactic cosmic rays faces at least two major problems. The first one is connected with the diffusion mechanism and was already discussed in the previous section 2.2. The second one is connected with the acceleration mechanism of CRs and will be discussed below. Here we shall address only the contradictions of the SM of GCR with observations, demonstrating that in most cases they are only apparent. Numerous subdominant phenomena, like re-acceleration, which in fact may affect the results in a noticeable way, will be omitted.

As far as diffusion is concerned, the problems arise due to the fast increase of the diffusion coefficient with energy $D(E) \propto E^{\mu}$, which results at high energy in a large 


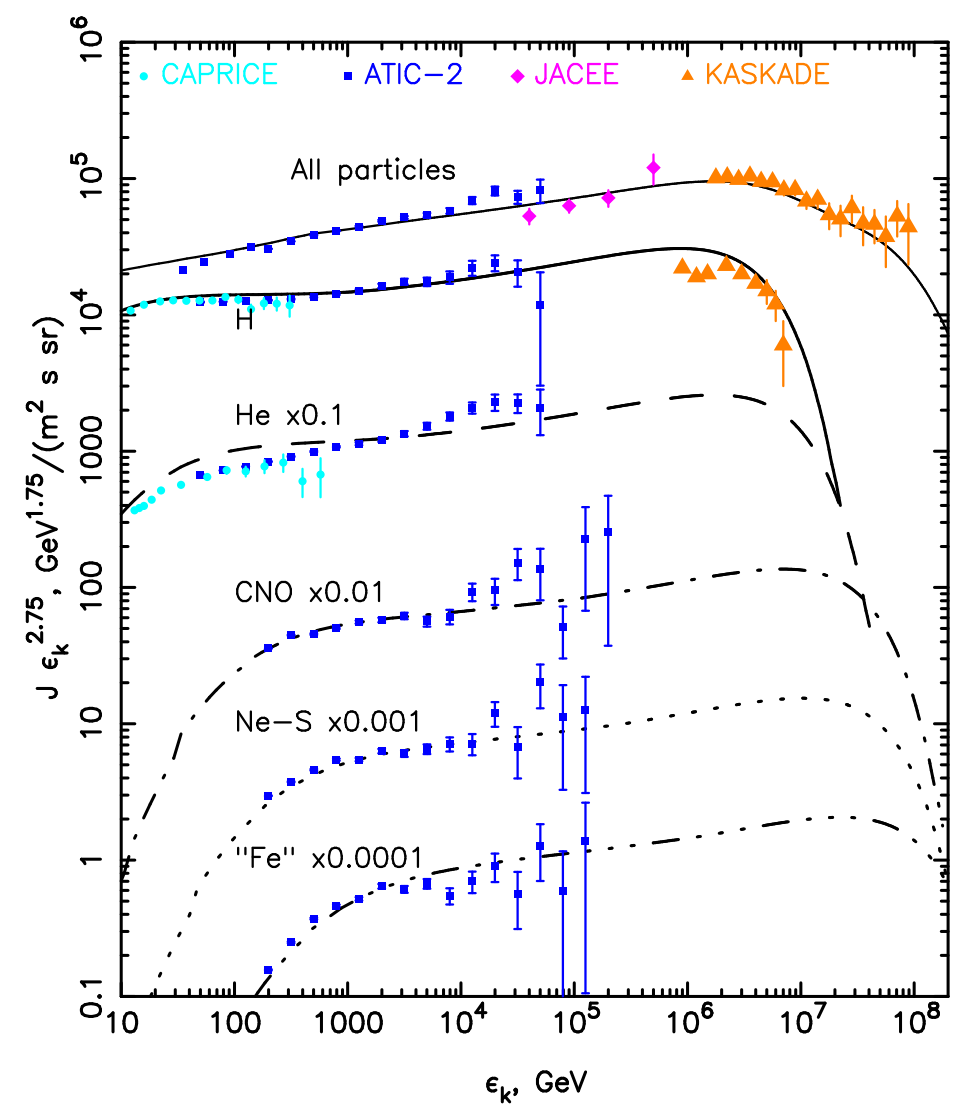

Figure 1. Fluxes and spectra calculated within the Standard Model [42] for all particles, protons and nuclei. Fluxes are shown as function of kinetic energy $\epsilon_{k}$ per nucleon. They are compared with the data of CAPRICE, ATIC-2, JACEE and KASCADE. The position of the knees for all nuclei are given by $\epsilon_{\mathrm{kn}} \approx 3 Z \mathrm{PeV}$. The end of Galactic spectrum is given by the iron knee $\epsilon_{k} \approx 80 \mathrm{PeV}$. At higher energies Galactic spectrum becomes very steep.

anisotropy, $\delta(E) \propto E^{\mu}$, and in a too small traversed grammage, $X_{\mathrm{cr}} \propto E^{-\mu}$. A possible solution was mentioned in section 2.2

It is interesting that another diffusion problem, the observation by PAMELA of the rise with energy in the $e^{+} / e^{-}$ ratio, may be solved within the SM [46] (see, however, the criticism of this model in 47]). The basic idea presented in [46] is the production of $e^{+} e^{-}$-pairs in pp-collisions in situ, i.e. at the shock front. Secondary positrons are immediately involved in the process of acceleration, in fact more efficiently than primary electrons, because they already have a power-law energy spectrum. As a result, the ratio $e^{+} / e^{-}$rises with energy. Of course, there were proposed other mechanisms of positron production, e.g. from pulsars [48, 49], from Dark Matter annihilation etc. (see [50] for review and references), but it is important that the positron excess can be successfully explained in the framework of the SM GCR.

The difference in spectrum slopes for protons and Helium was considered for a long time as a serious problem for shock acceleration, which is the most important component of the SM. This difference is presented in the most precise way by the PAMELA experiment [51]; the spectrum of Helium is harder. The slope difference is small, but statistically it is reliably provided, $\gamma_{p}-\gamma_{\mathrm{He}}=$ $0.1017 \pm 0.0014$.

Theoretically, the shock-wave acceleration does not distinguish among nuclei with equal rigidities. The predicted spectrum is a decreasing power-law with an exponent determined by the shock properties, i.e. the Mach number. However, according to PAMELA, spectra are not exactly power-law and the effective exponents are also different: 2.8 and 2.7 for protons and Helium, respectively. The understanding of these phenomena needs the inclusion of particle injection in the acceleration regime and the account of the escaping particles from the accelerator. We shall follow here the paper by Drury [52] with a clear physical analysis and the works 53, 54 for calculations.

In the standard approach the spectrum is calculated for particles accompanying the shock front, i.e. located within the acceleration length upstream and downstream. This spectrum differs from that of the escaping and captured particles. The latter drift downstream and are trapped there until the shock dies. They suffer adiabatic energy losses and do not contribute to the HE part of the observed spectrum. For escaping particles the exit model must be specified. In section 2.1 we considered the exit mechanism from 37]: at each time $t$ particles accelerated 
up to maximum energy $E_{\max }(t)$ freely escape from the shell. The total spectrum of escaping particles may be calculated by an integration over time. The highest energy particles escape at the beginning of the Sedov phase; the lower energy ones exit progressively later. The chemical composition changes with time because swept-up gas changes its chemical composition and ionization state. It means that the energy spectrum of nuclei with fixed ( $\mathrm{Z}$, A) is characterized by its own $\gamma_{g}$.

According to section 2.1, injection plays a similar role. An account for propagation through interstellar medium can also result in a difference in the spectral indices of protons and Helium.

\subsection{Beyond the SM: New component of the Galactic Cos- mic Rays?}

The observed knees are interpreted as the end of the Galactic CRs. Positions of knees define maximum acceleration energies, different for protons and nuclei, see Eq. (4). Though this picture looks now very natural, the way to its adoption was rather dramatic. Soon after the discovery of DSA 34 it was understood 55] that the maximum acceleration energy in this approach does not exceed $Z \times 10 \mathrm{TeV}$, much below the knee. Only later after the works by Bell [34], and Bell and Lucek 35], it was realized that an increasing of the magnetic field due to the Weibel instability may enhance the maximum energy up to the needed level $E_{\mathrm{Fe}}^{\max } \approx 0.1 \mathrm{EeV}$ given by Eq. (44). This story teaches us to be careful with assumptions about higher $E_{\max }$. Nevertheless, reasonable mechanisms to enhance $E_{\max }$ have been recently found.

The motivation for an additional component of the GCRs with $E_{\max }$ up to $10 \mathrm{EeV}$ is given by the interpretation of the ankle observed according to the latest data at $(3-4) \mathrm{EeV}$, as the transition from Galactic to extragalactic CRs at $E_{\mathrm{tr}} \sim(3-10) \mathrm{EeV}$. It was proposed in [56, 57, 58] that such an additional component may appear due to some unspecified sources different from SNR.

A plausible idea for increasing $E_{\max }$ is given by the explosion of SN in the stellar wind left behind by presupernova [59, 60]. The large magnetic field in the wind and the presence of heavy nuclei result in a larger $E_{\max }$. In particular in [59] this effect is maximized by considering the Wolf-Rayet stars as presupernovae.

In a more general way this idea was considered in 61. In this work an approximate relation between $E_{\max }$ and a SN total energy output $\mathcal{E}$ is obtained as $\log E_{\max } \approx$ $0.52 \log \mathcal{E}_{51}+2.1 \mathrm{TeV}$ for the protons, where $\mathcal{E}_{51}$ is the total energy output in units $10^{51} \mathrm{erg}$. The distribution of SNe over $E_{\max }$ is obtained using the distribution of SNe over $\mathcal{E}_{51}$. The high energy tail of the distribution gives sources with enhanced $E_{\max }$, and thus the diffuse spectrum at large $E$ extends up to $E \sim 1 \mathrm{EeV}$ for Iron nuclei.

In 62 a similar approach was used for the calculation of GCR diffuse flux from SNRs with large $E_{\max }$. SNRs were

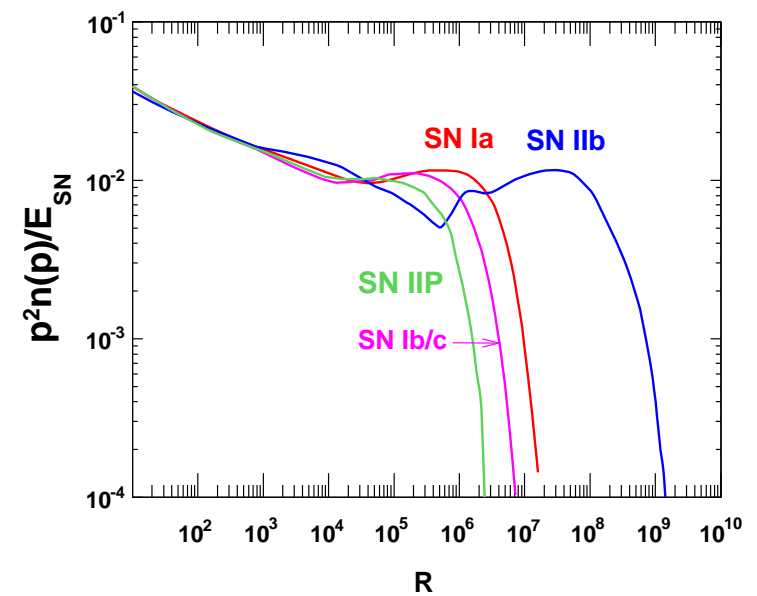

Figure 2. Diffuse spectra of Galactic CRs produced by four groups of SNRs, labeled as SN Ia, SN IIP, SN Ib/c and SN IIb (see [62]). Along the ordinate axis the plotted quantity is $p^{2} n(p) / E_{\mathrm{SN}}$, where $p$ is the particle momentum, $n(p)$ is proportional to the total number of accelerated particles with momentum $p$ produced by SN and $E_{\mathrm{SN}}$ is the total energy of SN explosion. Note that we generalized the original plot from 62] introducing dimensionless rigidity $R=p /\left(Z m_{N}\right)$ instead of dimensionless proton momentum $p / m_{N}$, as the authors recommended to do for nuclei. As seen from the plot, in the maximum of the curve the rigidity reaches $R_{\max }=3 \times 10^{7}$, i.e. $0.78 \mathrm{EeV}$ for Iron (see text).

subdivided into four groups according to the type of explosion: SNR Ia, SNR IIb/c, SNR IIP and SNR IIb. Each group of SNRs was characterized by fixed parameters of SN explosion and presupernova stellar wind, in which the shock propagates, accelerating particles. In Fig. 2 spectra of accelerated particles and $R_{\max }$ are shown for each calculated group. The group SN IIb represents a new HE component with a higher $E_{\max }$. This energy corresponds to the shock propagation through a presupernova stellar wind. For the evolution of SN IIb we use calculations published in 63. The presupernova of SN IIb is a red supergiant with mass $18 M_{\odot}$ on the Main Sequence. The stellar wind is presented by Helium $(Z=2)$ and at a smaller extent by Oxygen $(Z=8)$, since metals have very small abundances in presupernova stellar wind. The metals produced by SN explosion work as a piston, and the shock propagates ahead of this layer. Using Oxygen we obtain at the maximum of the curve $E_{\max }=Z R_{\max } \approx 0.2 \mathrm{EeV}$, and for Iron which has very low abundance $E_{\max } \approx 0.8 \mathrm{EeV}$, still below the ankle.

The next logical step in the discussion of SN explosions as sources of a new HE Galactic component is given by the hypernova explosion, a very rare (one per $10^{5}$ years in a galaxy) but very powerful event which can alone fill the Milky Way with cosmic rays of the observed density. Hypernovae are assumed to be the sources of GRBs. Such a model for GCR was considered in 64]. The explosion occurred $2 \times 10^{5}$ yr ago at distance about $500 \mathrm{pc}$ from the Sun. At present the highest energy particles passed the Sun, while the lower energy ones are still confined there. In this model the transition to extragalactic CRs occurs 
at $E \sim 0.2 \mathrm{EeV}$, too low for the "ankle transition".

An extreme case is considered in 65]. A sample of 1000 hypernovae are exploding randomly in the Milky Way with time interval $10^{5}$ years. They produce cosmic rays with a mass composition of $10 \%$ protons and $90 \%$ Iron nuclei with maximum energy $100 \mathrm{EeV}$. Protons, propagating rectilinearly, leave the Galaxy starting from $E \sim 1 \mathrm{EeV}$, while Iron nuclei diffuse in the Galactic magnetic fields and well explain the energy spectrum of the PAO starting from $10 \mathrm{EeV}$. At lower energy the spectrum and mass composition are explained as a mixture of protons and Iron nuclei. This is the case when hypernovae can explain the whole spectrum of UHECR at $E>2 \mathrm{EeV}$ by the Galactic CR component.

To conclude, the ankle model needs a new high energy Galactic component at $0.1 \mathrm{EeV} \lesssim E \lesssim 10 \mathrm{EeV}$ with a heavy mass composition. Though in principle it looks possible, for SNe such a case was not found. Probably other Galactic sources, such as pulsars 31], could fit this hope better.

\section{Extragalactic UHECR}

In this section the study of the transition will be proceeded moving from the end of the UHECR spectrum (from GZK cutoff in the case of protons) towards lower energies.

In the case of protons there are two spectral signatures of their propagation through CMB: pair-production dip, which is a rather faint feature caused by the pairproduction process given by Eq. (1), and a sharp steepening of the spectrum caused by pion photo-production process

$$
p+\gamma_{\mathrm{CMB}} \rightarrow \pi+X,
$$

called GZK cutoff [1, 2]. The GZK cutoff position is roughly defined by the energy where the pair-production energy loss in Eq. (1) and the pion production one of Eq. (14) become equal, namely at $E_{\mathrm{GZK}} \simeq 50 \mathrm{EeV}$ [12].

In the case of UHE nuclei the situation is different. For heavy enough nuclei $A>10$, taking into account only the CMB background, the steepening of the spectrum starts at the energy where adiabatic energy losses (due to the expansion of the universe) and losses due to pair production become equal (see energy losses in [66]), namely at $E \sim 20-50 \mathrm{EeV}$. The pair production process experienced by nuclei is affected only by the CMB radiation, while the photo-disintegration process takes place also on the Extragalactic Background Light (EBL) [67]. The latter is the process in which a nucleus with atomic mass number $A$ loses one or more nucleons interacting with the $\mathrm{CMB}$ or EBL:

$$
A+\gamma_{C M B, E B L} \rightarrow(A-n N)+n N
$$

The dominant process is one nucleon emission $(n=1)$ as discussed in 66]. The photo-disintegration of nuclei, to-



Figure 3. Modification factors for different generation indices $\gamma_{g}$.

gether with the pair production process, produces a steepening in the observed spectrum. The exact position of the flux suppression and its shape depend on the nuclei species as well as on the details of the cosmological evolution of the EBL which, in contrast to CMB, is not known analytically, being model dependent [67]. In general the steepening of the nuclei spectrum is not as sharp as the GZK and it occurs at lower energies 66.

Thus, the GZK cutoff is the most spectacular feature in the UHECR spectrum. However, it is valid only for UHE protons and the predicted shape of the GZK feature is model-dependent: it depends on a possible local over-density or deficit of sources and can be mimicked by low values of the maximum energy $E_{\max }^{\mathrm{acc}}$, that sources can provide, In contrast, the pair-production dip is practically model-independent mainly because protons contributing to this energy range arrive from distant cosmological sources.

\subsection{Pair-production dip}

Being a quite faint feature, the pair-production dip is not seen well in the spectrum presented as $\log J(E)$ versus $\log E$. It is more pronounced when analyzed in terms of the modification factor [12, 13, 15, 16]

$$
\eta(E)=J_{p}(E) / J_{p}^{\mathrm{unm}}(E)
$$

which is defined as the ratio of the spectrum $J_{p}(E)$, calculated with all energy losses taken into account, and the unmodified spectrum $J_{p}^{\text {unm }}(E)$, where only adiabatic energy losses are included.

Theoretical modification factors calculated for different source generation indices $\gamma_{g}$ are presented in Fig. 3. If one includes in the calculation of $J_{p}(E)$ only adiabatic energy losses, then, according to its definition, $\eta(E)=1$ (dashdot line). When the $e^{+} e^{-}$-production energy loss is additionally included, one obtains the $\eta(E)$ spectrum shown 

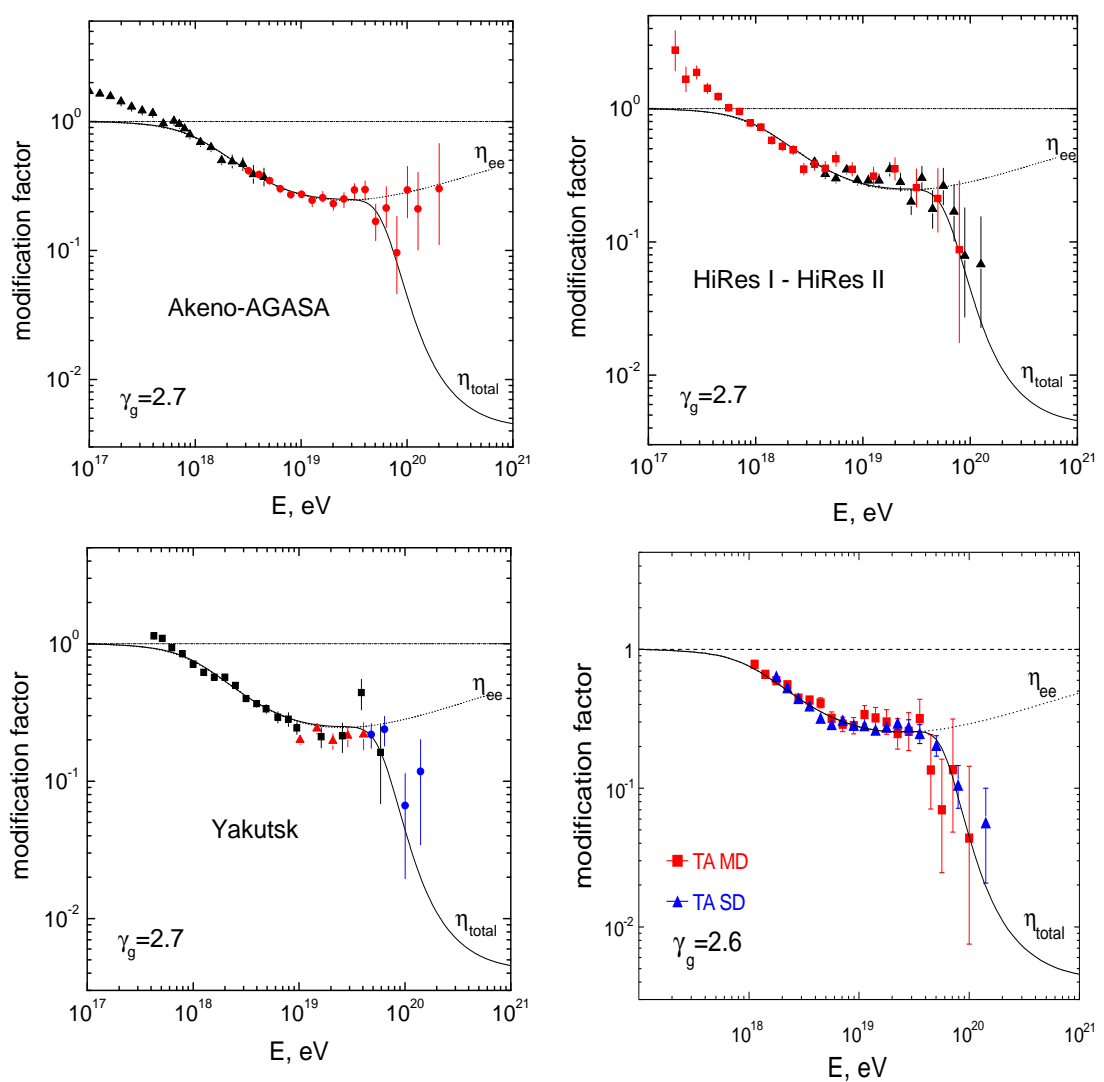

Figure 4. The predicted pair-production dip in comparison with Akeno-AGASA, HiRes, Yakutsk and Telescope Array (MD and SD) data [17]. All these experiments confirm the dip behavior with good accuracy, including also the data of Fly's Eye [17] (not presented here).

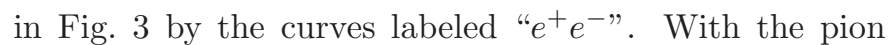
photo-production process being also included, the GZK feature (curves "total") appears. The observable part of the dip extends from the beginning of the GZK cutoff at $E \approx 40 \mathrm{EeV}$ down to $E \approx 1 \mathrm{EeV}$, where $\eta \approx 1$. It has two fattenings: one at energy $E_{a}^{\mathrm{tr}} \sim 10 \mathrm{EeV}$ and the other at $E_{b} \sim 1 \mathrm{EeV}$. The former automatically produces the ankle (see Fig. 4) and the latter provides an intersection of the flat extragalactic spectrum at $E \leq 1 \mathrm{EeV}$ with the steeper Galactic one. Since many non-essential factors in the numerator and denominator of Eq. (16) compensate or cancel each other, the dip in terms of the modification factor is a less model dependent physical quantity than the spectrum. In fact the dip is determined mostly by the interaction of protons with the CMB photons and it depends mainly on the CMB spectrum and the differential crosssection of $e^{+} e^{-}$pair-production. In particular it depends weakly on the spectral index of the generation spectrum. In Fig. 3 curves are plotted for $2.1 \leq \gamma_{g} \leq 3.0$ with a step $\Delta \gamma_{g}=0.1$, and uncertainties are seen as thickness of the curves.

Modification factors in Fig. 3 are presented for the case without cosmological evolution of the sources, which is usually described in the injection spectrum by a factor $(1+z)^{m}$ up to $z_{\max }$. The inclusion of evolution may noticeably change the modification factor, but in fact it al- lows to improve the agreement of the dip with data due to the additional free parameters $m$ and $z_{\max }$ (see Fig. 14 of Ref. [15]).

Thus, a remarkable property of the dip in terms of modification factor is its universality. The dimensionless quantity $\eta(E)$ remains the same with various physical phenomena being included in calculations [15, 16]: discreteness in the source distribution (distance between sources may vary from $1 \mathrm{Mpc}$ to $60 \mathrm{Mpc}$ ), different modes of propagation (from rectilinear to diffusive), local overdensity or deficit of sources, large-scale inhomogeneities in the sources distribution, some regimes of cosmological evolution of sources (most notably those observed for AGN) and fluctuations in the interactions. The phenomenon which modifies the dip significantly is the possible presence of more than $15 \%$ of nuclei in the primary radiation. Therefore, the shape of the proton dip in terms of modification factor is determined mostly by the interaction with the CMB.

Above the theoretical modification factor was discussed. The observed modification factor, according to definition, is given by the ratio of the observed flux $J_{\text {obs }}(E)$ and unmodified spectrum $J_{\text {unm }}(E) \propto E^{-\gamma_{g}}$, defined up to normalization as:

$$
\eta_{\mathrm{obs}} \propto J_{\mathrm{obs}}(E) / E^{-\gamma_{g}} .
$$

Here $\gamma_{g}$ is the exponent of the generation spectrum $Q_{\text {gen }}\left(E_{g}\right) \propto E_{g}^{-\gamma_{g}}$ in terms of initial proton energies $E_{g}$. 

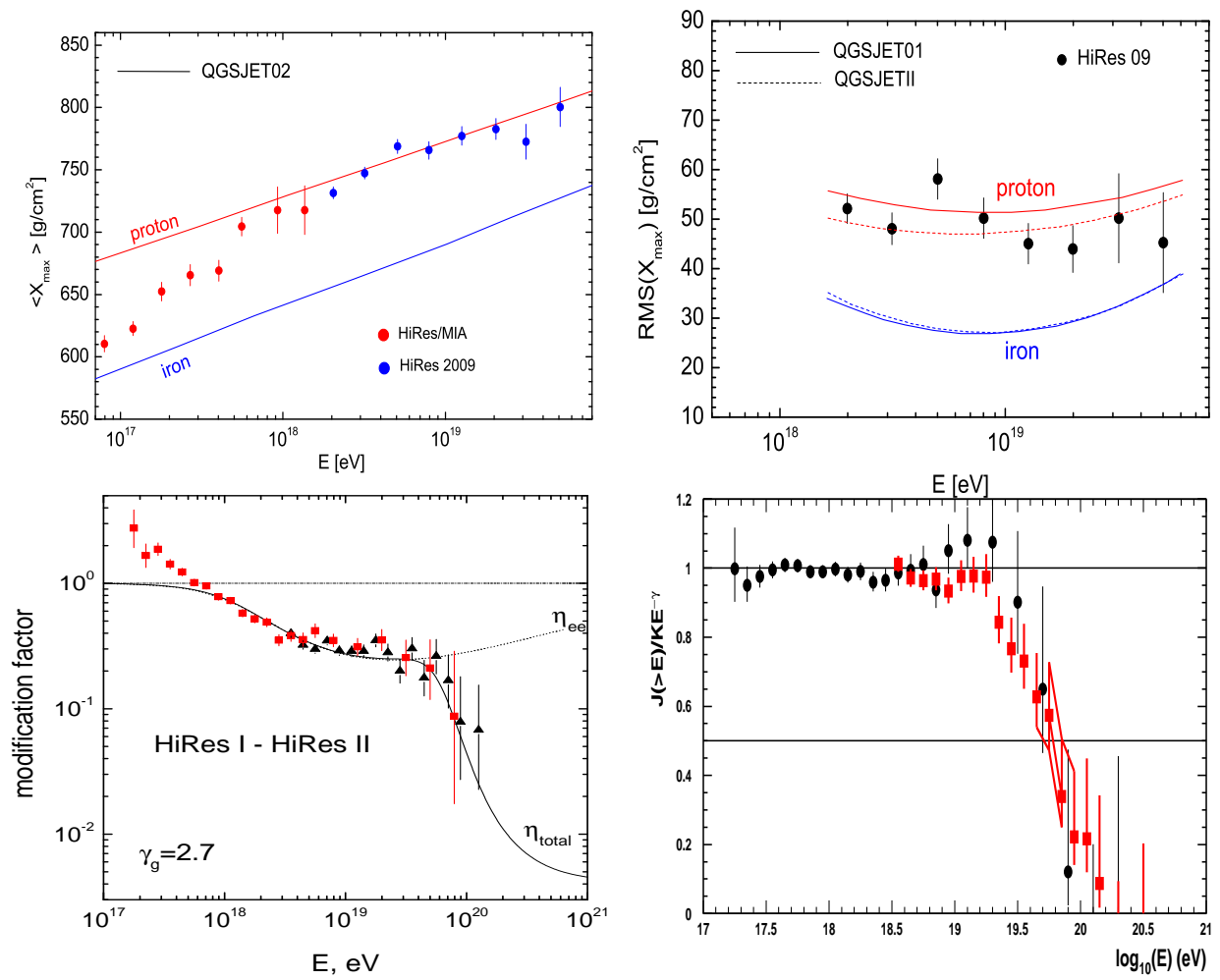

Figure 5. Mass composition and GZK cutoff as measured by the HiRes detector. In two upper panels $\left\langle X_{\text {max }}\right\rangle$ (left) and RMS (right) are presented as function of the energy. Both agree with a pure proton composition, shown by curves labeled 'proton'. The left-lower panel shows differential energy spectrum in terms of the modification factor. One can see a good agreement with the predicted shape of the GZK cutoff. The right-lower panel shows the quantity $E_{1 / 2}$ in the integral spectrum. This energy, a characteristic of the GZK cutoff, is found as $E_{1 / 2}=10^{19.73 \pm 0.07} \mathrm{eV}$ in good agreement with theoretical prediction $E_{1 / 2}=10^{19.72} \mathrm{eV}$ (see the text).

Fig. 4 shows that both the pair production dip and the beginning of the GZK cutoff up to $80 \mathrm{EeV}$ are well confirmed by experimental data of Akeno-AGASA, HiRes, Yakutsk and TA [17].

The comparison of the theoretical dip with observational data includes only two free parameters: exponent of the power-law generation spectrum $\gamma_{g}$ (the best fit corresponds to $\left.\gamma_{g}=2.6-2.7\right)$ and the normalization constant to fit the $e^{+} e^{-}$-production dip to the measured flux. The number of energy bins in the different experiments is $20-22$. The fit is characterized by $\chi^{2} /$ d.o.f. $=1.0-1.2$ for AGASA, HiRes and Yakutsk data. For this fit we used the modification factor without cosmological evolution of sources. As was explained above, using two additional evolutionary parameters, $m$ and $z_{\max }$, one can further improve the agreement.

In Fig. 4 one can see that at $E \lesssim 0.6 \mathrm{EeV}$ the experimental modification factor, as measured by Akeno and HiRes, exceeds the theoretical modification factor. Since by definition the modification factor must be less than one, this excess signals the appearance of a new component of cosmic rays at $E<E_{\mathrm{tr}} \approx 0.6 \mathrm{EeV}$, which can be nothing else but the Galactic cosmic rays. This interpretation is confirmed by the transition of a heavy component to protons in the upper-left panel, that with good accuracy occurred at the same energy. Thus, according to HiRes data the transition from extragalactic to Galactic cosmic rays, occurs at energy $E_{\mathrm{tr}} \sim 0.6 \mathrm{EeV}$ and is accomplished at $E \sim E_{b} \approx 1 \mathrm{EeV}$ (see left-upper panel in Fig. 5 as example).

\subsection{GZK cutoff.}

The two largest Extensive Air Shower (EAS) detectors, HiRes and Pierre Auger Observatory [3, 5], have observed a sharp steepening in the UHECR spectrum at $E \gtrsim(30-$ 50) EeV. Both collaborations claimed that the observed steepening is consistent with the GZK cutoff. But as a matter of fact, there is a dramatic conflict between these two results, which still leaves the problem open.

In this subsection we analyze the data of HiRes which provide a strong evidence in favor of the GZK cutoff. These data are supported also by the TA observations [4]. The data of PAO will be considered in the next subsection.

To interpret convincingly the spectrum steepening as the GZK cutoff one must prove that:

1. energy scale of the cutoff and its shape correspond to theoretical predictions,

2. the measured chemical composition is strongly dominated by protons.

Mass composition of the CR spectrum in turn may be measured with the help of 

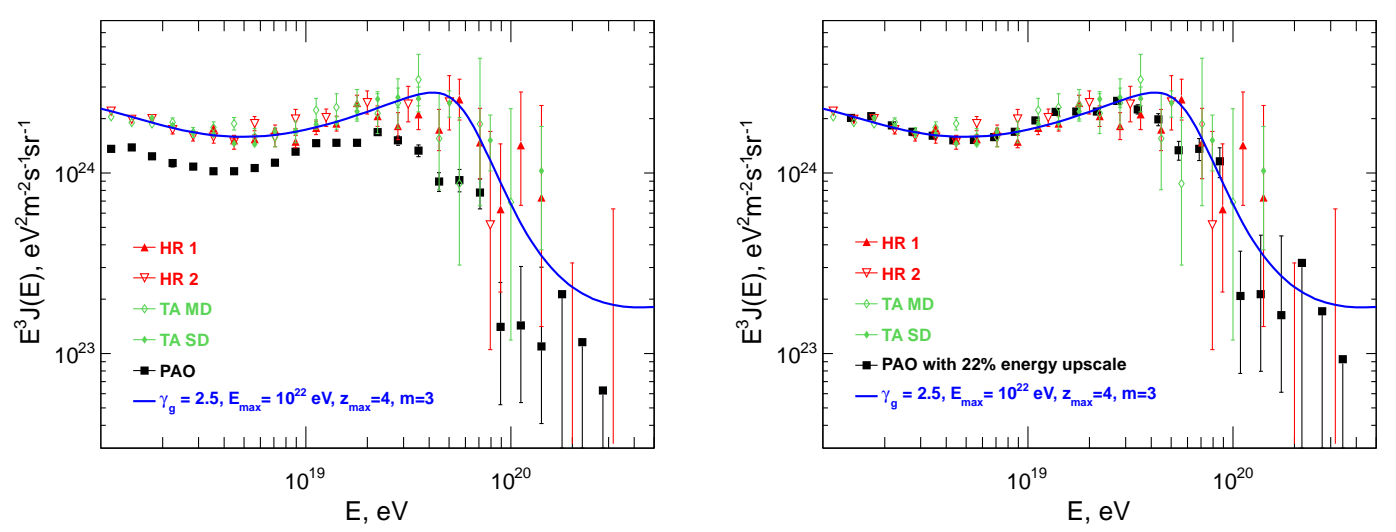

Figure 6. PAO spectrum [68, 69] (filled squares) compared with HiRes/TA spectra and with calculated spectrum (full line) with parameters indicated in the figures. In the left panel the spectra are shown with energy scales as determined in the experiments. In the right panel the data of all three experiments are recalibrated using the pair-production dip as a standard candle. For HiRes and TA the recalibration factor is 1.0, since these detectors describe well the dip. The PAO recalibration factor should be 1.22 to fit the dip shape. It is not trivial that after this recalibration the PAO flux coincides well with HiRes and TA fluxes. However, the contradiction of three PAO bins at $40-50 \mathrm{EeV}$ with the predicted GZK shape remains.
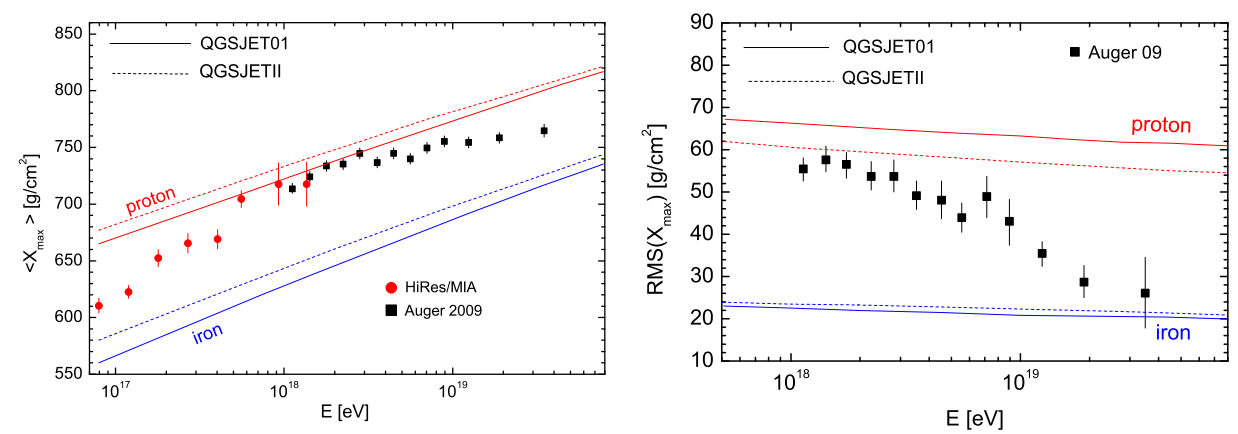

Figure 7. Auger data [0] for $\left\langle X_{\max }\right\rangle$ as function of the energy (left panel) and for $\operatorname{RMS}\left(X_{\max }\right)$, the width of the distribution over $X_{\max }$, (right panel). The calculated values for protons and Iron are given according to QGSJET01 [71] and QGSJETII [72] models. One can see from the right panel that RMS distribution becomes more narrow with increasing energy which implies a progressively heavier mass composition.

- $\left\langle X_{\max }\right\rangle(E)$, the average depth of atmosphere in $\mathrm{g} / \mathrm{cm}^{2}$, where a shower with energy $E$ reaches its maximum, and

- $\operatorname{RMS}\left(X_{\max }\right)$, which is the width of the distribution over $X_{\max }$.

These values measured by HiRes are displayed in Fig. 5 . From the left-upper panel of Fig. 5 one can see that the chemical composition changes from very heavy elements, probably Iron, at $E \sim 0.1 \mathrm{EeV}$, (data of HiRes-MIA 73]) to protons at $E \sim 1 \mathrm{EeV}$ (data of HiRes [17]). Such evolution fully corresponds to the SM for GCR (see Fig. 1). $\operatorname{RMS}\left(X_{\max }\right)$, a very sensitive tool for mass composition, also provides evidence for a proton-dominated composition at $E \gtrsim 1 \mathrm{EeV}$ and up to the highest energies (see upper-right panel of Fig. 5). Differential energy spectrum of the GZK feature in the modification factor form (leftlower panel) is in a reasonably good agreement with the theoretical prediction, though better statistics at higher energies is still needed for a final conclusion.

The integral energy spectrum of UHE protons, $J_{p}(>E)$, has another specific characteristic of the GZK cutoff, the energy $E_{1 / 2}$ [12]. It is based on the observation that the calculated integral spectrum below $50 \mathrm{EeV}$ is well approx- imated by a power-law function: $J_{p}(>E) \propto E^{-\tilde{\gamma}}$. At high energy this spectrum steepens due to the GZK effect. The energy where this steep part of the spectrum equals to half of its power-law extrapolation, $J_{p}(>E)=K E^{-\tilde{\gamma}}$, defines the value of $E_{1 / 2}$. This quantity is found to be practically model-independent; it equals to $E_{1 / 2}=10^{19.72} \mathrm{eV} \approx 52.5$ EeV [12]. Fig. 5 demonstrates how the HiRes collaboration found $E_{1 / 2}$ from observational data [74]. The ratio of the measured integral spectrum $J(>E)$ and the low-energy power-law approximation $K E^{-\tilde{\gamma}}$ was plotted as a function of energy. This ratio is practically constant in the energy interval $0.3-40 \mathrm{EeV}$, indicating that the power-law approximation is a good fit, indeed. At higher energy the ratio falls down and intersects the horizontal line 0.5 at the energy defined as $E_{1 / 2}$. It results in $E_{1 / 2}=10^{19.73 \pm 0.07} \mathrm{eV}$, in excellent agreement with the predicted value.

Thus, one may conclude that the HiRes data presented in Fig. 5 indicate the proton-dominated chemical composition and the presence of the GZK cutoff in both differential and integral spectra. The conclusion about proton composition is further supported by the recent TA data [17]. 

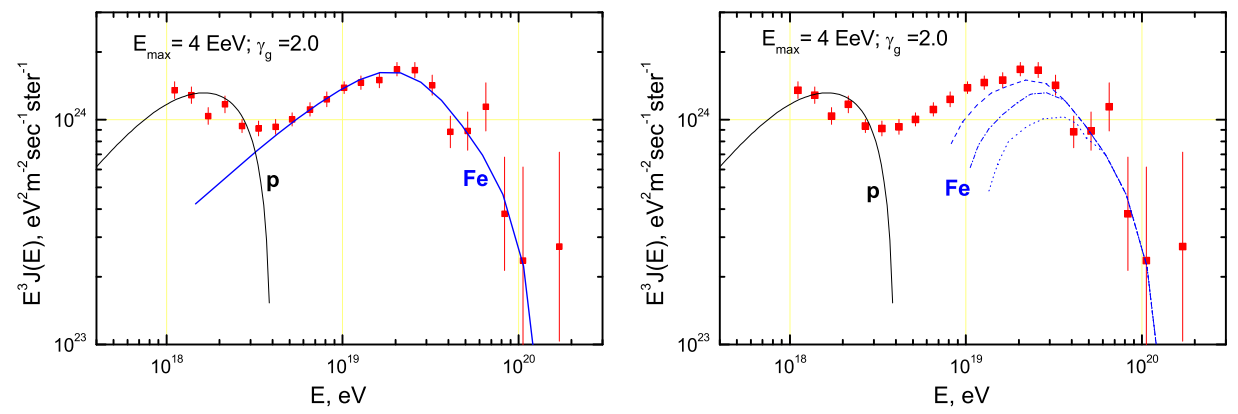

Figure 8. Left panel: Energy spectrum in the two-component model with protons and Iron nuclei for homogeneous distribution of sources and with $\gamma_{g}=2.0$ and $E_{\max }=4 \mathrm{EeV}$. Right panel: As in left panel but with a diffusion cutoff. The gap is expected to be filled by intermediate mass nuclei.

\subsection{PAO data: energy spectrum and mass composition}

The spectrum of the Pierre Auger Observatory, shown by filled squares in the left panel of Fig. 6, differs from the HiRes and Telescope Array spectra both in absolute normalization and in shape. However, one is allowed to shift the energy scale within systematic energy errors. Since the energy position of the pair-production dip is rigidly fixed by the interaction with CMB, in Ref. [15] and [16] it was proposed to use the dip as an energy calibrator ('standard candle'). The data of HiRes and TA have recalibration factors $k_{\text {cal }}=1.0$, since these data agree well with the energy position and shape of the pair-production dip (see left panel of Fig. 6). The Auger data (left panel of Fig. 6) disagree with the dip, but with the recalibration factor $k_{\text {cal }} \simeq 1.22$ a reasonable agreement can be reached. It is far non-trivial that simultaneously the Auger flux agrees well with the fluxes of HiRes and TA. This procedure was applied in 15, 16] imposing the condition of minimizing $\chi^{2}$ after recalibration of the data of AGASA, HiRes and Yakutsk detectors. A similar procedure based on the dip energy calibration was also used by Blümer et al. 11] for all experiments with recalibration factors $1.0,1.2,0.75$, and 0.625 for the HiRes, PAO, AGASA and Yakutsk detectors, respectively; a good agreement for all fluxes was demonstrated. This procedure was also successfully applied in 75].

The apparent coincidence of the PAO and HiRes/TA spectra is related to the low energy part of the energy spectrum in Fig. 6. At higher energies statistical uncertainties are too large to distinguish between the spectra.

While the HiRes and TA spectra are compatible with the GZK cutoff, the Auger spectrum is not. The steepening in the upscaled PAO spectrum starts at energy $E \lesssim 40$ $\mathrm{EeV}$, lower than $E_{\mathrm{GZK}} \simeq 50 \mathrm{EeV}$, and in three successive energy bins the PAO flux is significantly lower than one predicted for the GZK shape as shown in the right panel of Fig. 6] We could not reconcile the PAO cutoff shape with the GZK behavior by including in calculations different generation indices $\gamma_{g}$, evolution regimes, low acceleration maximum energy $E_{\max }$, local over-density of sources etc.

This disagreement is quite natural taking into account the mass composition measured by PAO (see Fig. 7), which dramatically differs from that of HiRes shown in
Fig. 5] The dependence of the $\operatorname{RMS}\left(X_{\max }\right)$ on energy, which steadily decreases and approaches the Iron value at $E \approx 35 \mathrm{EeV}$, is a strong example of this disagreement. Low RMS, i.e. small fluctuations, is a typical and reliable feature of a heavy nuclei composition. These data are further strengthened by other PAO measurements provided by surface detectors. They allow to extract two masscomposition dependent quantities: the atmospheric depth $\left\langle X_{\max }^{\mu}\right\rangle$, where muon-production rate reaches maximum, and maximum zenith angle $\theta_{\max }$ determined by the signal rise-time in surface Cerenkov detectors. Measurements of both quantities confirm the heavy mass composition and its dependence on energy obtained with the help of $\left\langle X_{\max }\right\rangle(E)$ and $\operatorname{RMS}\left(X_{\max }\right)$ [76] and 77]. The soon expected data on muon flux from the Auger Muon and Infill Ground Array (AMIGA) 78] will further clarify the mass composition.

An early steepening of the spectrum observed by the PAO can be explained as a property of nuclei propagation. However, it is a problem to explain simultaneously both spectrum and mass composition of PAO.

A wide class of mixed composition models 28 fails to fit the PAO mass composition at the highest energies. Most of them assume a source mass composition close to Galactic CRs, i.e. enhanced by protons. The observed mass composition in these models becomes heavier only at $E \gtrsim 50 \mathrm{EeV}$, when protons disappear due to the GZK cutoff [28]. In the paper [28], by Allard et al. (2008), an exceptional case of primaries being pure Iron nuclei was considered, though authors refer to this possibility as to an unnatural one. This model (see also [79] fig. 4 and [80]) explains the observed Auger energy spectrum.

Mass composition becoming progressively heavier with increasing energy appears in the rigidity-dependent acceleration, when the maximum attainable energy for a nucleus with charge number $Z_{i}$ is $E_{i}^{\max }=Z_{i} E_{p}^{\max }$ and the contribution of nuclei with charge $Z^{\prime}<Z$ to this energy disappears, while heavier nuclei with larger $Z$ exist. In this way the 'disappointing model' 81] was built.

The disappointing model is based on the following observations and assumptions:

- According to the HiRes (Fig. 5) and PAO (Fig. 7) 
data, the observed primaries at energy $(1-3) \mathrm{EeV}$ are predominantly protons.

- It is assumed that they have extragalactic origin.

- The particles at higher energies are extragalactic nuclei with charge number $Z$ steadily increasing with energy.

- Acceleration of CRs in sources is rigidity dependent, $E_{i}^{\max }=Z_{i} \times E_{p}^{\max }$.

It was demonstrated in 81] that to avoid a proton dominance at the highest energies, one must assume that the maximum energy of the accelerated protons is limited, $E_{p}^{\max } \lesssim(4-10) \mathrm{EeV}$. This conclusion is valid for a large range of generation indices, $\gamma_{g} \sim 2.0-2.8$, and for a wide range of cosmological evolution parameters. The calculated proton and nuclei energy spectra for $\gamma_{g}=2.0$, $E_{\max }=Z \times 4 \mathrm{EeV}$ and without cosmological evolution are shown in Fig. 8. In the left panel the two component model (protons and Iron) is presented. In the right panel of Fig. 8 intermediate primary nuclei are included in the framework of the diffusive propagation through intergalactic magnetic fields (see [81] for details). The rigidity dependent acceleration, $E_{i}^{\max }=Z_{i} \times E_{p}^{\max }$, provides a steadily increasing charge number $Z$ as energy increases.

The disappointing model as it is presented here and in [81] is not complete. The energy spectra must be calculated in diffusive approximation for different distances between sources. Energy dependence of $\left\langle X_{\max }\right\rangle(E)$ and $\operatorname{RMS}\left(X_{\max }\right)$ must be consistent with calculated energy spectrum.

This model was called 'disappointing' because of lack of many signatures predicted in proton-dominated models, such as cosmogenic neutrino production and correlation of CR arrival directions with distant sources.

Recently a similar model 82], based on the rigiditydependent acceleration, was proposed for making the chemical composition heavier with energy increasing. This model uses the reasonable assumption [83, 16] that the space density $n_{s}$ of UHECR sources decreases with increasing of the maximum acceleration energy. The idea may be explained in the following way.

Consider accelerators which produce protons $(p)$ and nuclei $(i)$ with maximum energy $E_{p}^{\max }$ and $E_{i}^{\max }=Z_{i} \times$ $E_{p}^{\max }$, respectively. The space density of sources is assumed to depend on $E_{\max }$,

$$
n_{s}\left(E_{p}^{\max }\right) \propto\left(E_{p}^{\max }\right)^{-\beta} .
$$

Generation rate of protons with energy $E$ per unit space volume is proportional to

$$
Q_{p}(E) \propto \int_{E} d E_{p}^{\max }\left(E_{p}^{\max }\right)^{-\beta} \propto E^{-\beta+1},
$$

because sources with $E_{p}^{\max }<E$ do not contribute. For nuclei $i$

$$
Q_{i}(E) \propto \int_{E / Z_{i}} d E_{p}^{\max }\left(E_{p}^{\max }\right)^{-\beta} \propto Z_{i}^{\beta-1} E^{-\beta+1}
$$

(sources with $E_{p}^{\max }<E / Z_{i}$, i.e. $Z_{i} E_{p}^{\max }<E$, do not contribute). Then from Eqs. (18) and (19)

$$
Q_{i}(E) / Q_{p}(E) \propto Z_{i}^{\beta-1} .
$$

Eq. (20) shows that the production of nuclei is enhanced by a factor $Z_{i}^{\beta-1}$; it can affect the mass composition calculations in the mixed composition models.

\section{Three transition models}

In this section we discuss three models of transition from Galactic to extragalactic CRs: ankle, dip and mixed composition models. One feature is common for all of them: the transition is described as an intersection of a steep Galactic spectrum with a more flat extragalactic one. The agreement with the SM for GCR (see subsection 2.1) is one more criterion which these models have to respect. According to the Standard Model, the benchmark of the end of GCR is given by the Iron knee at energy $E_{\mathrm{Fe}} \approx 80 \mathrm{PeV}$ (see Fig. 1) and at $E>E_{\mathrm{Fe}}$ it has an exponential cutoff.

Motivated by the interpretation of the ankle as the transition to extragalactic CR at $E_{a}^{\mathrm{tr}} \sim(3-10) \mathrm{EeV}$, one has to assume [56, 57, 58] an additional component of GCR accelerated up to energies much beyond the Iron knee (see subsection 2.4).

Observational data which have the power either to confirm or to reject each transition model include energy spectrum, elongation curve $\left\langle X_{\max }\right\rangle(E), \operatorname{RMS}\left(X_{\max }\right)$ and anisotropy. Below these models will be discussed in the historical succession of appearance: ankle, dip and mixed composition.

\subsection{Ankle model}

This is the traditional model based on the interpretation of the ankle as the spectrum feature where transition occurs (see e.g. [22] - 23]). In fact, this is a very natural model since transition occurs because the extragalactic component is very hard. This component is assumed to have a pure proton composition with a flat generation spectrum $Q_{\text {extr.p }} \propto E^{-2}$ valid for non-relativistic shock acceleration. Energy losses modify the spectrum insignificantly at $E \lesssim 40 \mathrm{EeV}$. The beginning of the ankle at $E_{a}^{\mathrm{obs}} \sim(3-4) \mathrm{EeV}$ corresponds to the energy where fluxes of Galactic and extragalactic CRs get equal. Thus, the Galactic CRs should be presented by an additional component accelerated up to energy at least by factor 30 - 40 times higher than the maximum energy in the Standard Model. In the majority of the ankle models, e.g. [56, 57, 86, 23], the large fraction of the observed cosmic rays has a Galactic origin at $E \gtrsim 10 \mathrm{EeV}$. To facilitate the acceleration problem one should assume a heavy-nuclei composition of the new component.

The transition at the ankle is illustrated by the right panel of Fig. 9. The curve "extr.p" presents the calculated extragalactic flux of protons and the dash-dot line gives the Galactic CR spectrum. The latter is obtained 

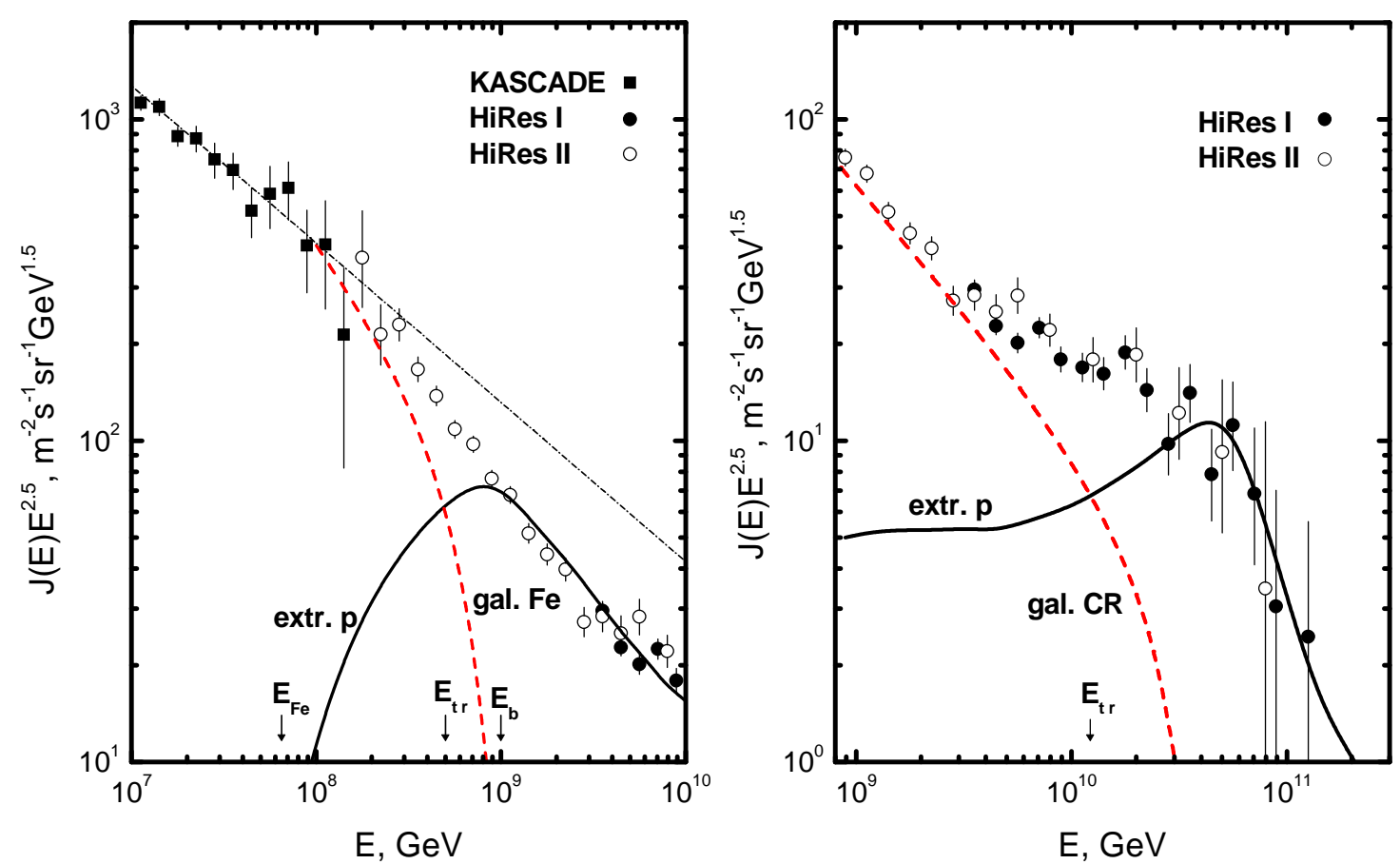

Figure 9. Transition in the dip (left panel) and ankle (right panel) models. In both cases solid lines give the calculated extragalactic proton spectrum and dashed lines describe the Galactic iron spectrum. $E_{\mathrm{tr}}$ is the energy of intersection of the Galactic and extragalactic spectra and $E_{\mathrm{Fe}}$ gives the position of the iron knee. $E_{b} \approx 1 \mathrm{EeV}$ in the left panel is the energy where the transition from Galactic to extragalactic CRs is completed.

by subtracting the extragalactic component from the total observed flux following the procedure first suggested in [87]. The observed dip in the spectrum may be explained by the Hill-Schramm's mechanism [18].

Another problem of the ankle model is the contradiction with the measured average depth of EAS maximum, $\left\langle X_{\max }\right\rangle(E)$, in the energy range $(1-5) \mathrm{EeV}$. While all data, including both HiRes and PAO, show proton or light nuclei composition here, the ankle model needs a heavy Galactic component, predicting too small $\left\langle X_{\max }\right\rangle(E)$ in contradiction with observations (see the right panel of Fig. 10 and right panel of Fig. 4 in [88]). This contradiction is found also in [28].

The ankle at an energy higher than $3 \mathrm{EeV}$ contradicts also the anisotropy calculated in [89].

\subsection{Dip model}

The dip model is based on the assumption that UHECR at $E \gtrsim 1 \mathrm{EeV}$ are mostly extragalactic protons. This assumption is confirmed by the observation of the pairproduction dip in the energy range $(1-40) \mathrm{EeV}$ and the beginning of the GZK cutoff in the energy range (40 100) $\mathrm{EeV}$ (see Fig. 4). Both features are signatures of a proton dominated spectrum. As was discussed above the shape of the dip allows an admixture of light nuclei, though not more than $15 \%$. The transition from Galactic to extragalactic CRs occurs as the intersection at $E_{\text {tr }} \sim$ $0.5 \mathrm{EeV}$ of the steep Galactic component (dashed line in the left panel of Fig. 9) with the flat extragalactic proton component shown by 'extr.p' curve (this curve appears to be falling down since the spectrum is multiplied by $E^{2.5}$ ). The flatness of the extragalactic spectrum is provided by the distribution of sources over the maximum acceleration energy $n_{s}\left(E_{\max }\right) \propto E_{\max }^{-\beta}($ see below) or, in the case of diffusive propagation, by the 'magnetic flattening' 16, 90, 91]. The transition is completed at energy $E_{b} \approx 1 \mathrm{EeV}$, i.e. it occurs at a visible feature in the $\mathrm{CR}$ spectrum known as the 'second knee', at energy between $E_{\mathrm{tr}}$ and $E_{b}$ in the left panel of Fig. 9

The basic features of the dip model are as follows 14, 15, 16]:

- The primary flux is strongly proton-dominated.

- Sources are e.g. AGN 92 with a neutron mechanism for particle escape [93, 94] which provides a pure proton generation spectrum. Another case of enhancement in the proton fraction at relativistic shock acceleration is given in [16].

- To reproduce the observed shape of the dip, indices for the generation rate per unit co-moving volume $Q(E) \propto E^{-\gamma_{g}}$ in models without evolution are to be $\gamma_{g}=2.6-2.7$. Such steep spectra can be obtained for the case of usual injection $q(E) \propto\left(E^{-2}-E^{-2.3}\right)$ from a single source assuming sources distribution over 

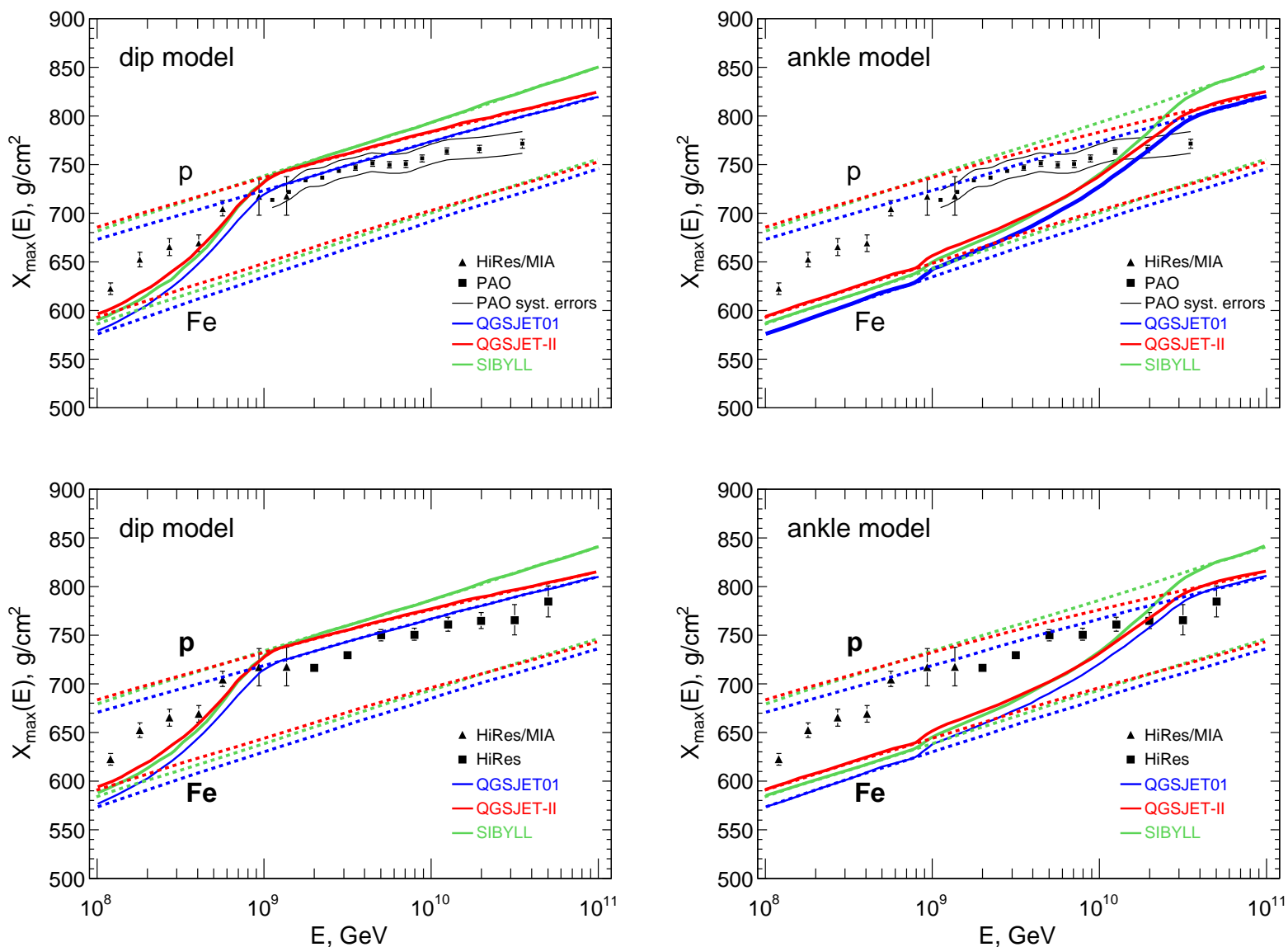

Figure 10. The calculated elongation curves $\left\langle X_{\max }\right\rangle(E)$ for the dip model (two left panels) and ankle model (right panels). The calculated curves $\left\langle X_{\max }\right\rangle(E)$ are shown by the thick solid lines for QGSJET01 71] model of interaction, by the thick dashed lines for QGSJET-II 72], and by dotted lines for SIBYLL [84]. The data points are measurements of HiRes-Mia (filled triangles), HiRes and PAO (both filled boxes). The PAO data in upper panels with systematic errors, shown by the thin curves, are taken from [85]. The lines 'p' and 'Fe' present the elongation curves for proton and Iron which are used for calculations of the model elongation curves in each panel. For PAO data they are theoretical curves, for HiRes they include cuts, detector's properties etc. and are taken from curves 'iron' and 'proton' in the upper-left panel of Fig. 5 As main result of these plots one may notice the great discrepancy of the ankle model with the data.

maximum acceleration energies $n_{s}\left(E_{\max }\right) \propto E_{\max }^{-\beta}$ [83, 16], where $n_{s}$ is the space density of sources.

- The generation index $\gamma_{g}=2.6-2.7$ is the main factor which provides the agreement of proton spectrum with the pair-production dip and the GZK cutoff (see subsection 3.1). The cosmological evolution of the sources can be easily included, affecting mostly the low energy part of the spectrum. Inclusion of additional parameters allows to improve the fit. In particular, in 15] the spectrum was calculated with an account for cosmological evolution of AGN as it follows from X-ray observations. The calculated spectrum shown in Fig. 14 of Ref. [15] is in excellent agreement with data.

- The mode of propagation (from rectilinear to diffusive) is important in two energy ranges: at low energies $E \lesssim 1 \mathrm{EeV}$ and at $E \gtrsim 60 \mathrm{EeV}$. In both regimes the distance between sources becomes important in the diffusive regime. In the low-energy range it makes the spectrum at $E<E_{\mathrm{tr}}$ more flat, "magnetic flattening" shown by curve 'extr.p' in Fig. 9. And at $E \gtrsim 60 \mathrm{EeV}$ the GZK shape becomes more steep in the case of large distance between sources (see [95] [96]).

The confirmation of the dip model follows from: $(i)$ the agreement of the dip energy spectrum shape (see Fig. 4 and Fig. 6) with observations, (ii) the agreement of fluxes of all experiments after the dip-based energy recalibration (see Fig.66and Refs. [16], Blümer et al. from [1]], 75]), (iii) the agreement of $\left\langle X_{\max }\right\rangle(E)$ with the bulk of observational data in the left panel of Fig. 10, excluding only the highest energy PAO experimental points.

A characteristic feature of the dip model is the sharp transition from Galactic Iron to extragalactic protons. It results in a steep increase of the $\left\langle X_{\max }\right\rangle(E)$ with energy at $E$ below $1 \mathrm{EeV}$ (see left panels of Fig. 10). This feature differentiates the dip model from the ankle model, where 
the increase of $\left\langle X_{\max }\right\rangle(E)$ is less steep (see the right panel of Fig. 10). The difference is due to less hard proton spectrum at $E \lesssim 1 \mathrm{EeV}$ in the ankle model, see Fig. 9.

The key observation to accept or reject the dip model is the chemical composition of UHECR at $E>1 \mathrm{EeV}$. In the case of a substantial admixture of nuclei in the spectrum (>20\%) the dip model should be rejected.

At present the dip model is confirmed by the data of HiRes by strong proton-dominance at $E>1 \mathrm{EeV}$, and is in contradiction with the Auger measurements of $\left\langle X_{\max }\right\rangle(E)$ and RMS at $E>4 \mathrm{EeV}$.

\subsection{Mixed composition model}

The main concept of the mixed composition model (see Allard et al. [28], [97], 98]) is based on the argument that any acceleration mechanism operating in gas involves different nuclei in acceleration process and thus the primary flux must have a mixed composition.

The basic features of the mixed composition model are as follows [28, 97, 98]:

- It is assumed that the Galactic component of CRs above $0.1 \mathrm{EeV}$ has a pure iron composition while the extragalactic CRs have a mixed-nuclei composition. Transition can occur either at ankle or below it, down to the second knee. Its position is parameterdetermined.

- In its basic variant 28], the source composition of extragalactic CRs is assumed to be almost the same as for Galactic CRs, with protons and Helium being the dominant components. However, a pure species composition is also possible in the framework of this model, in particular pure Iron, for understanding the physical effects.

- The source energy spectra are taken as power-law $Q(E) \propto E^{-\gamma_{g}}$ with a generation index $\gamma_{g}=2.1-2.3$. The maximum acceleration energy is assumed to be rigidity dependent with a maximum energy for nuclei $i$ being $E_{i}^{\max }=Z_{i} E_{p}^{\max }$, where $Z_{i}$ is the nucleus atomic number. The maximum energy of protons is typically taken $E_{p}^{\max }=300 \mathrm{EeV}$.

- The cosmological evolution of sources is described by the factor $k_{\mathrm{ev}}=(1+z)^{m}$ for a wide range of regimes: $m=0$ in the absence of evolution, $m=3$ like for star formation evolution and $m=4$ for a strong cosmological evolution. These regimes are typically considered only for small redshifts $z \lesssim 1$ or 1.3 and for larger $z$ the frozen evolution with $k_{\mathrm{ev}}=$ const up to $z_{\max }$ is assumed.

- The sources are assumed to be distributed homogeneously in the universe, so that the propagation mode in extragalactic magnetic fields, from rectilinear to diffusive, does not influence the energy spectrum at observation. The propagation is studied using a Monte Carlo approach with the inclusion of pair-production energy losses and nuclei photodisintegration on $\mathrm{CMB}$ and EBL.

At a first glance one may expect that the large number of free parameters, such as generation index, parameters of cosmological evolution and coefficients of source nuclei composition, can provide a broad variety of observed mass compositions and spectra. However, as it was demonstrated in [28], the predictions are very much constrained due to photo-disintegration of nuclei on EBL and CMB radiations. The basic physics phenomena and their results are as follows.

Generically in the mixed models the mass composition becomes lighter at $E>10 \mathrm{EeV}$, because intermediate and heavy nuclei are destroyed by the EBL photons while protons survive. In principle this situation may change only above $50 \mathrm{EeV}$, when GZK cutoff in the proton spectrum appears, while heavy nuclei, e.g. Iron, are still not photodisintegrated by the CMB photons and may dominate. In realistic cases the dominant component in mixed models are protons.

In the academic case of a fixed nuclei species at the source (Allard et al (2008) in 28]) secondary protons are the dominant component at all energies if the primaries are light or intermediate mass nuclei, and only in the case of Iron the protons are subdominant. It is interesting to note that because of an efficient destruction of light and intermediate nuclei the two-component model with only proton and Iron injected at sources gives a reasonable agreement with observations 81].

In the mixed models the large primary proton component contributes more than the secondary protons, strengthening further effect of proton dominance. Therefore, the GZK feature is also present in the mixedcomposition models.

The proton dominance in mixed models is illustrated by Fig. 11. In the left panel decomposition of the diffuse flux in the elemental components is presented. The dominant component is primary protons, and the next subdominant is secondary protons and Helium. In the right panel the elongation curve $\left\langle X_{\max }\right\rangle(E)$ is presented with data points from HiRes-MIA, HiRes-stereo and Fly's Eye superimposed. Starting from $E \geq 3 \mathrm{EeV}$ the elongation curve $\left\langle X_{\max }\right\rangle(E)$ tends to a proton-dominated composition in accordance with HiRes and Fly's Eye data. In contrast, the PAO data tend at the highest energies to an Iron-dominated composition.

Transition from Galactic to extragalactic component in the mixed models depends on the choice of parameters. In most models transition occurs at the ankle, see Allard et al. in [28]. However, in the conceptually important paper by Allard, Olinto, Parizot (2007) from [28] it was emphasized that for strong source evolution and flat generation spectra the intersection of Galactic and extragalactic components occurs between $0.5 \mathrm{EeV}$ and $1 \mathrm{EeV}$, i.e. at the second knee, as in the dip model. The transition in this 



Figure 11. Left panel: Diffuse spectrum decomposed in its elemental components, calculated in the mixed-composition model by Allard, Olinto, Parizot (2007) from [28]. The proton dominance seen in the spectrum is a generic prediction of the mixed composition models. Right panel: Elongation curve $\left\langle X_{\max }\right\rangle(E)$ in the same model as in the left panel. The mass composition evolves from almost a pure iron composition at $E \approx 0.3 \mathrm{EeV}$ to a lighter composition due to an enrichment by protons and light nuclei of extragalactic origin. At energy $E_{a}=3 \mathrm{EeV}$ the transition to pure extragalactic component is completed and chemical composition evolution proceeds further due to photo-disintegration of nuclei. At energy $E \approx 13 \mathrm{EeV}$ all nuclei are disappearing faster than before and composition becomes strongly proton-dominated at $E \geq 30 \mathrm{EeV}$.

model is shown in Fig. 12 The transition begins at $2 \mathrm{EeV}$ and is accomplished at $3 \mathrm{EeV}$.

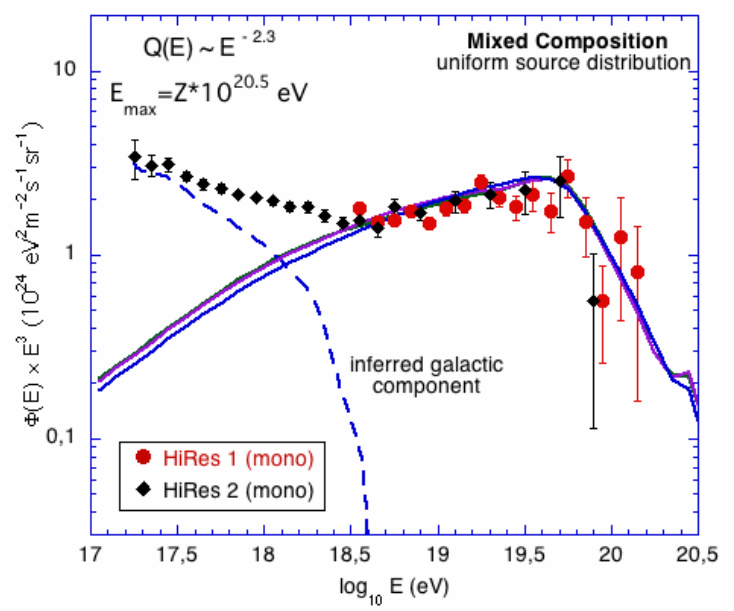

Figure 12. Diffuse propagated spectrum for mixed model by Allard et al. (2007) [28] with generation index $\gamma_{g}=2.3$ and cosmological evolution $(1+z)^{3}$ up to $z=1.3$ and with a frozen evolution at larger $z$ up to $z_{\max }=6$. The transition accomplishes at $E \approx 3 \mathrm{EeV}$, i.e. below the ankle. At highest energies the spectrum is dominated by protons (see right panel of Fig. 11) and shows the GZK cutoff, compared in the figure with HiRes data.

The dominance of protons (as an example see Fig. 11) was the reason why the $\left\langle X_{\max }\right\rangle(E)$ predicted by mixed composition models is in a better agreement with HiRes data than with PAO data. The observations of PAO show that mass composition becomes heavier with increasing energy, and thus the existing calculations in the framework of mixed models agree better with the HiRes data.

However, with the recent proposal [82], discussed in subsection 3.3. the power of the mixed composition model for fitting the PAO data may change due to a possible en- hancement of the heavy nuclei production.

\section{Discussion and Conclusions}

The transition from Galactic to extragalactic CRs takes place in the energy interval $(0.1-10) \mathrm{EeV}$. and it gives a key for understanding both galactic and extragalactic cosmic rays.

In its low-energy part this range includes the highenergy tail of the Galactic CRs. The information obtained at these energies on the Galactic CRs involves the maximum energy of acceleration, the chemical composition, the anisotropy and the mode of propagation in the Galaxy. It may unveil the whole picture of the origin of the Galactic CRs.

The low-energy part of the extragalactic cosmic rays spectrum can give a key information on the existence of the pair-production dip and on the propagation of the CRs in extragalactic magnetic fields.

Therefore, the experimental studies in the transition region $(0.1-10) \mathrm{EeV}$ are of paramount importance in this field of research, with the mass composition measured by different methods being probably the most important task.

There are four working detectors which cover partially the above-mentioned region: KASCADE-Grande 99], Tunka 100], Yakutsk 75] and IceTop/IceCube 101]. There are also projects to extend the observations of Telescope Array and PAO to low energies, $(0.1-1) \mathrm{EeV}$ at TALE [102] and at LE-Auger [103]. The Auger detector has a great potential to explore the low-energy region of the UHECR spectrum. At present there are already two new detectors at $\mathrm{PAO}$ collecting data at this energy; HEAT [104], High Elevation Auger Telescope, detecting the fluorescent light at higher elevation angles; and AMIGA [78], Auger Muons and Infill Ground Array, for the detection of the EAS muon component. 
These detectors, together with TALE, Tunka, Yakutsk and IceCube/IceTop will provide information on all radiations from EAS, including fluorescent and Cherenkov light, muons and radio radiation. One may expect that in this way the present controversy between the mass compositions in the HiRes and Auger detectors will be unambiguously solved. The recent measurement [76, 77] of the muon-production depth $X_{\max }^{\mu}$ and maximum zenith angle $\theta_{\max }$ by on-ground Auger detectors is an important step in this direction.

At the low-energy end of the UHECR the energy spectra are measured with an unprecedented accuracy for cosmic ray physics. However, in fact even in experiments with the same technique, like HiRes and Auger, the energy scales are different due to systematic errors (see left panel of Fig. 6). Such difference is more natural for different techniques, e.g. scintillator and fluorescent ones in TA detector. The problem of the energy scale hopefully may be solved combining all observational methods. However, there is a physical 'standard candle' for the detector energy calibration, given by the fixed energy position of the pairproduction dip. The recalibration factor $k_{\text {cal }}$ can be found by the spectrum shift to the energy at which the agreement between observed and predicted dips is the best [15, 16]. If the observed dip is really caused by pair-production process, the fluxes measured in all experiments must coincide. This is what we see indeed in Fig. 6, in [15, 16, 75] and in Blümer at al. [11].

Since the mass composition is expected to be different for Galactic and extragalactic CRs, its measurement gives a reliable way to test the transition. The best method at present to determine the $\mathrm{CR}$ chemical composition is given by measuring $\left\langle X_{\max }\right\rangle(E)$. Unfortunately this method suffers from many uncertainties, including those in the value of fluorescent yield, absorption of UV light in the atmosphere and uncertainties in the interaction models, needed to convert the mass composition into the observed $\left\langle X_{\max }\right\rangle(E)$. Systematic errors in the $\left\langle X_{\max }\right\rangle$ measurement can be as large as $20-25 \mathrm{~g} / \mathrm{cm}^{2}$, to be compared with the difference of about $100 \mathrm{~g} / \mathrm{cm}^{2}$ between the $\left\langle X_{\max }\right\rangle(E)$ for protons and Iron. A better sensitivity to distinguish different nuclei is given by the width of the $\left\langle X_{\max }\right\rangle(E)$ distribution, i.e. $\operatorname{RMS}\left(X_{\max }\right)$ [88].

The most self-consistent conclusions on the nature of the observed UHECRs are obtained at present for HiRes detector: it has observed both signatures of proton propagating through CMB; namely pair-production dip and the GZK cutoff. Moreover, the conclusion about protons as primaries is confirmed by the direct measurements of proton composition with the help of $\left\langle X_{\max }\right\rangle(E)$ and RMS. GZK cutoff is also found in the integral spectrum with characteristic energy $\log E_{1 / 2}=19.73 \pm 0.07$, in excellent agreement with theoretical prediction $\log E_{1 / 2}=19.72$. Good agreement with the dip energy shape and with $E_{1 / 2}$ allow to conclude that the HiRes basic energy scale is correct. HiRes data agrees well with the dip model with AGN as the sources and with cosmological evolution of AGN taken from X-ray observations.

The Auger data are quite different. Measurements of mass composition using $\left\langle X_{\max }\right\rangle(E)$ and RMS and confirmed by independent data of muon-production depth $X_{\max }^{\mu}$ and rise-time of the signal in water-Cerenkov tanks indicate nuclei as primaries with steadily increasing $\langle A\rangle$ starting from $4 \mathrm{EeV}$ and up to $35 \mathrm{EeV}$, where mass composition practically reaches pure Iron. The Auger mass composition, if confirmed, excludes the dip model and the observed high-energy steepening as GZK cutoff. At present there is no nuclei-based model which explains simultaneously the Auger energy spectrum $J(E)$ and elongation curve $\left\langle X_{\max }\right\rangle(E)$.

There are three models of transition: ankle, dip and mixed composition one. They differ most notably by the energy of transition and by the mass composition of the extragalactic component.

The ankle model was the first one which appeared in literature. It was proposed already in 1960s after the discovery of a flat feature, the ankle, at the Volcano Ranch array [20]. The transition in this model is described in the most natural way, as the intersection of a steep $\left(\propto E^{-3.1}\right)$ Galactic heavy-nuclei component (Iron) with a flat extragalactic proton component with a generation spectrum $\propto E^{-2}$. The transition, as calculated in different models, occurs at $E_{\mathrm{tr}} \sim(3-10) \mathrm{EeV}$. To fill the gap between $0.1 \mathrm{EeV}$ (Iron knee) and $E_{\text {tr }}$ indicated above the ankle models need a new yet unknown high energy Galactic component beyond the Standard Model for Galactic Cosmic Rays. Recently the ankle model has been strongly disfavored by $\left\langle X_{\max }\right\rangle$ measured below the ankle (see right panel of the Fig. 10) due to small $X_{\max }$ predicted by the model. It is also disfavored by the large (not observed) anisotropy predicted at $E \sim(1-3) \mathrm{EeV}$ [89].

The dip model is based on the pair-production dip induced in the extragalactic spectrum of protons interacting with the CMB radiation. The dip is well confirmed by the observations of HiRes, TA, Yakutsk and AGASA (see Fig. (4). The successful energy calibration of all observations (see Fig. 6 as an example) using the fixed energy position of the pair-production dip can be considered as another (indirect) evidence in favor of the dip model.

The transition in the dip model occurs as an intersection of the steep Iron Galactic spectrum with a very flat extragalactic spectrum at energy $(0.1-1) \mathrm{EeV}$. The flatness of this spectrum can be provided as a 'magnetic flatness' in the case of diffusive propagation 90, 91] or due to the sources distribution over $E_{\max }[83,16]$. A robust prediction of the dip model is the proton-dominated mass composition above $E \gtrsim 1 \mathrm{EeV}$. It is confirmed by the HiRes and TA data; the calculated elongation curve $\left\langle X_{\max }\right\rangle(E)$ is in good agreement with the bulk of data (see left panel of Fig. 10). However, it strongly contradicts the highest energy Auger data, especially the $\operatorname{RMS}\left(X_{\max }\right)$, presented in the right panel of Fig. [7. The Auger - HiRes controversy on the mass composition is a crucial point for the survival of the dip model. 
In the mixed composition models the presence of various nuclei is assumed in the generation spectrum of the extragalactic component. This assumption is very reasonable, because various nuclei are present in the gas where acceleration process operates. Mixed composition models with a wide choice of parameters are considered in [28]. They include different generation indices, cosmological evolution and most notably different relative abundances of nuclei at generation. However, as was explained in subsection 4.3. a basic feature common to most mixed composition models is the proton dominance at the highest energies $E \gtrsim 10 \mathrm{EeV}$. This feature is in conflict with the Auger data at these energies that shows the mass composition getting progressively heavier with energy increasing and reaching Iron at $E \approx 35 \mathrm{EeV}$. On the other hand, the mixed composition models fit reasonably well the HiRes and Fly's Eye data. The Galactic spectrum at the highest energies is assumed to be Iron dominated. The transition energy is a model-dependent quantity, being determined by $\gamma_{g}$ and the parameters chosen for sources cosmological evolution. However, for the flat generation indices $\gamma_{g}=2.1-2.3$ and a reasonable choice of the cosmological evolution, the transition energy is about $3 \mathrm{EeV}$ (as an example see Fig. 12). The elongation curve shown in the right panel of Fig. 11 changes the slope at approximately the same energy, and the mass composition evolves towards the pure proton composition. The calculated elongation curve in Fig. 11 agrees well above the transition with the data of HiRes stereo and Fly's Eye, though nowadays the data of HiRes agrees better at $E \gtrsim 3 \mathrm{EeV}$ with a pure proton composition.

Much better quality of data on the $\left\langle X_{\max }\right\rangle(E)$ is needed to distinguish the dip and mixed-composition models by $X_{\max }$ measurements.

With the recent proposal 82] for an enhancement of heavy nuclei production at the sources, the mixedcomposition models may hopefully describe better the Auger elongation curve $\left\langle X_{\max }\right\rangle(E)$ and its RMS.

As short conclusion one may state that while the ankle models of transition are severely disfavored, mostly by the $\left\langle X_{\max }\right\rangle(E)$ data at $E \sim 10^{18} \mathrm{eV}$, the dip and mixed composition models are in a good agreement with the $\left\langle X_{\max }\right\rangle(E)$ data from the Fly's Eye, HiRes and TA detectors. The present problem is the conflict of data on mass composition between the HiRes and TA on one side, and the PAO on the other. We do expect that problems with mass composition in the energy range $(0.1-3) \mathrm{EeV}$, of fundamental importance to asses the transition issue, will be soon resolved with the HEAT, AMIGA, KASCADE-G, TALE, Tunka, Yakutsk and IceCube/IceTop experiments.

\section{Acknowledgments}

We are grateful to Johannes Knapp and Sergey Ostapchenko for very useful discussions and to the Pierre Auger Collaboration for permission to use their data [68, 69, 85] prior to journal publication. We thank the anonymous Referee for a careful reading of the paper and numerous valuable questions and corrections. The work of V.B. was partly supported by the Ministry of Science and Education of Russian Federation (agreement 8525).

\section{References}

[1] K. Greisen, Phys. Rev. Lett. 16, 748 (1966).

[2] G. T. Zatsepin, and V. A. Kuzmin, Pisma Zh. Experim. Theor. Phys. 4, 114 (1966).

[3] R. U. Abbasi et al. Phys. Rev. Lett. 100, 101101 (2008); arXiv:astro-ph/0703099

H. P. Dembinski for the Pierre Auger Collaboration, arXiv:astro-ph/1107.4809.

[4] C. C. H. Jui [Telescope Array Collab.], arXiv:1110.0133, Y.Tsunesada [Telescope Array Collab.], arXiv:1111.2507

[5] J. Abraham et al., Phys. Rev. Lett. 101, 06110.1 (2008).

[6] F. W. Stecker, Phys. Rev. 180, 1264 (1969).

[7] V. S. Berezinsky, and G. T. Zatsepin, Sov. Journ. Nucl. Phys. 13, 453 (1971).

[8] V. S. Berezinsky, S. I. Grigorieva, and G. T. Zatsepin, Proc. 14th ICRC (Munich 1975) 2, 711 (1975); Astrophys. Sp. Sci. 36, 17 (1975).

[9] M. Hillas, Proc. 14th ICRC (Munich 1975) 2, 717 (1975).

[10] J. L. Puget, and F. W. Stecker, Proc. 14th ICRC (Munich 1975) 2, 734 (1975).

[11] P. Bhattacharjee, and G. Sigl, Phys. Rep. 327, 109 (2000); M. Nagano, and A. A. Watson, Rev. Mod. Phys. 72, 689 (2000); A. Letesier-Selvon, and T. Stanev, Rev. Mod. Phys. 83, 907 (2011), arXiv: 1103.0031;

K. Kotera, and A. V. Olinto, Ann. Rev. Astron. Astrophys. 49 (2011), arXiv:1101.4256

F. Aharonian, A. Bykov, E. Parizot, V. Ptuskin and A. Watson, Space Science Reviews 16697 (2012), arXiv:1105.0131

J. Blümer, R. Engel, and J. R. Hörandel, Progress in Part. and Nucl. Phys. 63, 293 (2009);

U. Katz, and Ch. Spiering, Prog.Part.Nucl.Phys. 67651 (2012), arXiv:1111.0507

[12] V. S. Berezinsky, and S. I. Grigorieva, Astron. Astrophys. 199, 1 (1988).

[13] T. Stanev et al, Phys. Rev. D 62, 093005 (2000).

[14] V. Berezinsky, A. Z. Gazizov, and S. I. Grigorieva, Phys. Lett. B 612, 147 (2005); astro-ph/0502550

[15] V. Berezinsky, A. Z. Gazizov, and S. I. Grigorieva, Phys. Rev. D 74, 043005 (2006); hep-ph/0204357

[16] R. Aloisio, V. Berezinsky, P. Blasi, A. Gazizov, S. Grigorieva, and B. Hnatyk, Astropart. Phys. 27, 76 (2007); astro-ph/0608219

[17] R. U. Abbasi et al. [HiRes Collab.], Phys. Rev. Lett. 92, 151101 (2004);

V. P. Egorova et al. [Yakutsk Collab.], Nucl. Phys. B (Proc. Suppl.) 3, 136 (2004);

K. Shinozaki et al. [AGASA Collab.], Nucl. Phys. B (Proc. Suppl.) 3, 151 (2006);

M. Honda et al. [Akeno Collab.], Phys. Rev. D 70, 525 (1993); D. J. Bird et al. [Fly's Eye Collab.], Astrophys. J. 424, 491 (1994);

G. B. Thomson [Telescope Array Collab.], arXiv:1010.5528,

C. C. H. Jui [Telescope Array Collab.], arXiv:1110.0133.

M. Roth [Pierre Auger Collab.], astro-ph/0706.2096;

L. Perrone [Pierre Auger Collab.], astro-ph/0706.2643.

[18] C. T. Hill, and D. N. Schramm, Phys. Rev. D 31, 564 (1985).

[19] S. Yoshida, and M. Teshima, Progr. Theor. Phys. 89, 833 (1993).

[20] J. Linsley, Proc. 8th ICRC (Jaipur 1963) 477 (1963).

[21] The Pierre Auger Collaboration, Phys. Lett. B 685, 239 (2010); The Pierre Auger Collaboration, ICRC2011, arXiv:1107.4809

[22] D. De Marco, and T. Stanev, Phys. Rev. D 72, 081301 (2005).

[23] E. Waxman, Nucl. Phys. B (Proc. Suppl.) 87, 345 (2000). 
[24] M. Nagano et al. [Akeno Collab.], J. Phys. G: Nucl. Part. Phys. 18, 423 (1992);

D. J. Bird et al. [Fly's Eye Collab.], Phys. Rev. Lett. 71, 3401 (1993);

A. V. Glushkov et al. [Yakutsk Collab.], JETP Lett. 73, 115 (2001).

[25] D. R. Bergman, and J. W. Beltz, J. Phys. G 34, 359 (2007), arXiv:0704.3721

[26] G. Kulikov, and G. Khristiansen, JETP 35, 635 (1958).

[27] W. D. Apel et al. [KASCADE-Grande Collab.], Phys.Rev.Lett. 107171104 (2011), arXiv:1107.5885

[28] D. Allard et al., Astron. Astrophys. 443, L29 (2005), astro-ph/0505566

D. Allard, E. Parizot, and A. V. Olinto, Astropart. Phys. 27, 61 (2007);

D. Allard et al. J. Phys. G 34, 359 (2007), astro-ph/0512345

D. Allard et al. JCAP 0810:033, (2008), arXiv:0805.4779

C. De Donato, and G. A. Medina-Tanko, Astropart. Phys. 32, 253 (2009).

[29] W. Baade, and F. Zwicky, Proc. of National Acad. of Scien. of the United States 20, 259, 1934; Phys. Rev. 46, 76 (1934).

[30] V. L. Ginzburg, Usp. Fiz. Nauk. (Sov. Phys. Usp.) 51, 343 (1953);

V. L. Ginzburg, and Syrovatsky, The Origin of Cosmic Rays, Pergamon Press, Oxford (1964).

[31] V. S. Berezinsky, S. V. Bulanov, V. A. Dogiel, V. L. Ginzburg, and V. S. Ptuskin, Astrophysics of Cosmic Rays, North Holland, Amsterdam, 534 p. (1990).

[32] A. W. Strong, I. V. Moskalenko, and V. S. Ptuskin, Ann. Rev. of Nucl. and Part. Science 57, 285 (2007).

33] P. Blasi, arXiv:1012.5005

[34] G. F. Krymsky, Sov. Phys. Dokl. Acad. Nauk USSR 243, 1306 (1977);

W. I. Axford et al. Proc. 15th ICRC, Plovdiv 11, 132 (1977);

A. R. Bell, Mon. Not. RAS 182, 147 (1978);

R. D. Blandford, and J. P. Ostriker, Astrophys. J. 221, L229 (1978).

[35] A. R. Bell, and S. G. Lucek, MNRAS 321, 433 (2001); A. R. Bell, MNRAS 353, 550 (2004)

[36] E. Amato, and P. Blasi, MNRAS 371, 1251 (2004); E. Amato, and P. Blasi, MNRAS Lett. 364, 76 (2005).

[37] V. S. Ptuskin, and V. N. Zirakashvili, Advances in Space Research 37, 1898 (2006).

[38] D. Ellison, L. O .C. Drury, and J.-P. Meyer, Astrophys. J. 487, 197 (1997).

[39] J.-P. Meyer, L. O .C. Drury, and D. Ellison, Astropart. Phys. J. 487, $182(1997)$

[40] E. G. Berezhko, and L. T. Ksenofontov, J. Exp. Theor. Phys. 89, 391 (1999)

[41] D. De Marco, P. Blasi, and T. Stanev, JCAP 0706027 (2007), arXiv:0705.1972

[42] E. G. Berezhko, and H. J. Völk, arXiv:0704.1715

[43] For a detailed discussion see the papers by V. Ptuskin, and L. O'C. Drury in this topical issue.

[44] P. Blasi, and E. Amato, JCAP 1201010 (2012), arXiv:1105.4521

P. Blasi, and E. Amato, JCAP 1201011 (2012), arXiv: 1105.4529

[45] O. Adriani et al. [PAMELA Collab.], Nature 458, 607 (2009), arXiv:08010.4995

[46] P. Blasi, Phys. Rev. Lett. 103, 051104 (2009).

[47] M. Kachelrieß, S. Ostapchenko, and R. Tomas, Astrophys. J. 733, 119 (2011).

[48] F. Aharonian, A. Atoyan, and H. J. Völk, Astron. Astroph. 294, L41 (1995).

[49] M. Pohl, Phys. Rev. D 79, 041301 (2009).

[50] P. D. Serpico, arXiv:1108.4827

[51] O. Adriani et al. [PAMELA Collab.], Science 332, 69 (2011); arXiv:1103.4055

[52] L.O'C. Drury, arXiv:1009.4799.

[53] M. A. Malkov, P. H. Diamond, and R. Z. Sagdeev,
Phys.Rev.Lett. 108081104 (2012), arXiv:1110.5335

[54] Y. Ohira, and K. Ioka, arXiv:1011.4405

55] P. O. Laggage, and C. J. Cesarsky, Astron. Astrophys. 125, 249 (1983).

[56] A. M. Hillas, J. Phys. G. Nucl. Part. Phys. 31, R95 (2005).

[57] A. M. Hillas, arXiv:0607109.

[58] T. Gaisser, Invited talk at XVI Int. Symp. on Very High Energy Cosmic Ray Interactions, 2010, Batavia, USA; arXiv:1010.5996

[59] H. J. Völk, and P. L. Biermann, Astrophys. J. 333, L65 (1988).

[60] V. S. Berezinsky, and V. S. Ptuskin, Astron. Astrophys. 215, 399 (1989).

[61] L. G. Sveshnikova, Astron. Astrophys. 409, 799 (2003).

[62] V. Ptuskin, V. Zirakoshvili, and E-S. Seo, Astrophys.J. 71831 (2010), arXiv: 1006.0034.

[63] T. Nozawa et al. Astrophys. J. 713, 356 (2010).

[64] S. D. Wick, C. D. Dermer, and A. Atoyan, Astropart. Phys. 21, 125 (2004).

[65] A. Calvez, A. Kusenko, and S. Nagataki, Phys. Rev. Lett. 105, 091101 (2010), arXiv:1004.2535

[66] R. Aloisio, V. Berezinsky, and S. Grigorieva, Astrop. Phys. in press DOI: 10.1016/j.astropartphys.2012.07.010, arXiv:0802.4452 [astro-ph];

R. Aloisio, V. Berezinsky and S. Grigorieva, Astrop. Phys. in press DOI: 10.1016/j.astropartphys.2012.06.003, arXiv:1006.2484 [astro-ph.CO].

[67] F. W. Stecker, M. A. Malkan, and S. T. Scully, Astrophys. J. 648, 774 (2006); Astrophys. J. 658, 1392 (2007);

T. M. Kneiske, T. Bretz, K. Mannheim, and D.H. Hartmann, Astron. Astrophys. 413, 807 (2004);

A. Franceschini, G. Rodighiero and M. Vaccari, Astron. Astrophys. 487, 837 (2008)

[68] P. Abreu et al. [Pierre Auger Collab.], arXiv:1107.4809

$69]$ F. Salamida [Pierre Auger Collab.], 32nd ICRC, Beijing, China, 2011.

70] J. Abraham et al. [Pierre Auger Collab.], Phys. Rev. Lett. 104 091101 (2010);

J. A. Bellido et al [Pierre Auger Collab.], Proc. 31st ICRC Lodz 2009

[71] N. N. Kalmykov, and S. S. Ostapchenko, A. I. Pavlov, Bull. Russ. Acad. Sci. Phys. 58, 1966 (1994); Nucl. Phys. Proc. Suppl. 52B, 17 (1997).

72] S. Ostapchenko, Phys. Rev. D 83, 014018 (2011); Phys. Rev. D 74, 014026 (2006); Nucl. Phys. Proc. Suppl. 151 B, 143 (2006).

[73] R. U. Abbasi et al. [HiRes-MIA Collab.], Phys. Lett. B 556, 1 (2003)

[74] R. U. Abbasi et al. [HiRes Collab.], Phys.Rev.Lett. 100101101 (2008), arXiv:astro-ph/0703099

[75] A. A. Ivanov, S. P. Knurenko, and I. E. Sleptsov, New Journ. Phys. 11, 065008 (2009).

[76] D. Garcia-Gamez, for the Pierre Auger Collaboration, arXiv:astro-ph/1107.4804

[77] L. Gazon, for the Pierre Auger Collaboration, J.Phys.Conf.Ser. 375052003 (2012), arXiv:astro-ph/1201.6265.

[78] F. Sanchez, for the Pierre Auger Collaboration, J.Phys.Conf.Ser. 375052006 (2012), arXiv:astro-ph/1107.4807.

79] D. Allard, arXiv:astro-ph/1111.3290.

[80] A.M. Taylor, M. Ahlers, F.A. Aharonian, Phys. Rev. D84 105007 (2011), arXiv:astro-ph/1107.2055.

[81] R. Aloisio, V. Berezinsky, A. Gazizov, Astropart. Phys. 34, 620 (2011);

J. Phys. Conf. Ser. 337, 012042 (2012).

[82] C. Blaksley, E. Parizot, Astropart. Phys. 35, 342 (2012).

[83] M. Kachelrieß, and D. Semikoz, Phys. Lett. B 634, 143 (2006).

[84] R. S. Fletcher, T. K. Gaisser, P. Lipari, and T. Stanev, Phys. Rev. D 50, 5710 (1994).

[85] P. Abreu et al. [Pierre Auger Collab.], arXiv:1107.4804

[86] T. Wibig, and A. W. Wolfendale, J. Phys. G 31, 255 (2005).

[87] V. Berezinsky, S. Grigorieva, and B. Hnatyk, Astropart. Phys. 22, 617 (2004).

[88] R. Aloisio, V. Berezinsky, P. Blasi, S. Ostapchenko, Phys. Rev. D 77, 025007 (2008) 
[89] G. Giacinti, M. Kachelriess, D. V. Semikoz, and G.Sigl, JCAP 1207031 (2012), arXiv:1112.5599

[90] M. Lemoine, Phys. Rev. D 71, 083007 (2005).

[91] R. Aloisio, and V. Berezinsky, Astrophys. J. 625, 249 (2005).

[92] V. Berezinsky, A. Gazizov, and S. Grigorieva, arXiv:astro-ph/0210095

[93] V. S. Berezinsky, Invited lecture at 15th ICRC (Plovdiv, Bulgaria) 10, 84 (1977)

[94] A. Atoyan, and C. D. Dermer, New Astron. Rev. 48, 381 (2004), astro-ph/0402646

[95] R. Aloisio, and V. Berezinsky, Astrophys. J. 612, 900 (2004).

[96] V. Berezinsky, and A. Gazizov, Astrophys. J. 669, 684 (2007).

[97] D. Allard, A. V. Olinto, and E, Parizot, astro-ph/0703633

[98] N. Globus, D. Allard, and E. Parizot, arXiv:0709.1541

[99] K.-H. Kampert [KASCADE Grande Collab.], Nucl. Phys. B. (Proc. Suppl.) 122 C, 422 (2003).

[100] B. V. Antokhonov et al. Texas Symp. on Relativistic Astroph., Heidelberg, Germany, POS (Texas 2010) 138.

[101] Ice Cube Collab., Proc. 32 st ICRC Beijing 2011 arXiv:1111.2735 2 [astro-ph.HE].

[102] D. R. Bergman [TA/TALE Collab.], Proc. of 29th ICRC (Pune) v. 8, 141 (2005); ibid C. C. H. Jui v. 8, 101 (2005).

[103] M.C. Medina, M. Gomez Berisso, I. Allekotte, A. Etchegoyen, G. Medina Tanco, A.D. Supanitsky, Nucl.Instrum.Meth. A566 302 (2006), astro-ph/0607115

[104] T. H. J. Mathes [Pierre Auger Collab.], J.Phys.Conf.Ser. 375 052006 (2012), arXiv:1107.4807. 